\title{
VARIABILITÉ SPATIALE DES TEMPÉRATURES DANS LE CONTEXTE DU CHANGEMENT CLIMATIQUE A L'ÉCHELLE DU VIGNOBLE DE MARLBOROUGH (NOUVELLE-ZÉLANDE)
}

\author{
Félix PHILIPPE ${ }^{12}$, Andrew STURMAN ${ }^{2}$ et Hervé QUENOL ${ }^{3}$ \\ ${ }^{1}$ Institut de Géographie Alpine, Université Grenoble Alpes (UJF) \\ 14bis, avenue Marie reynoard, 38100 Grenoble - France \\ felixphilippe@sfr.fr \\ ${ }^{2}$ Centre for Atmospheric Research \\ Department of Geography, University of Canterbury \\ Private Bag 4800 Christchurch, Nouvelle Zélande \\ andrew.sturman@canterbury.ac.nz \\ ${ }^{3}$ UMR6554 LETG-Rennes-COSTEL (IFR90 CAREN/OSUR) \\ Université Rennes 2, Place du Recteur Henri Le Moal \\ 35043 Rennes Cedex - France \\ herve.quenol@uhb.fr
}

\begin{abstract}
Résumé
Ce travail a pour objectif de mettre en place une méthodologie de mesures (météorologiques et agronomiques) et de modélisation spatiale du climat adaptée aux échelles fines, afin d'étudier la variabilité spatiale du climat dans les régions viticoles dans le contexte du changement climatique. La région de Marlborough, première région productrice de vin en Nouvelle-Zélande, fait l'objet d'investigations du fait des forts enjeux associés à la pérennité de l'activité viti-vinicole au niveau local, régional et national. Une première analyse des températures sur une période récente, menée à partir de stations météorologiques du réseau national et de deux réseaux locaux d'enregistrement de la température, permet de mettre en évidence une forte variabilité spatiale des températures à une échelle régionale et surtout locale. L'attention est notamment portée sur l'occurrence des gelées ainsi que sur les valeurs de différents indices bioclimatiques utilisés en viticulture. Par la suite sont analysées les premières projections réalisées à échelle fine pour le moyen terme (2030-2049) et la fin du siècle (2080-2099), afin d'apporter des premiers éléments de réponse quant aux futurs changements climatiques à l'échelle de la région de Marlborough.
\end{abstract}

Mots-clés : viticulture, changement climatique, climat local, Marlborough, Nouvelle-Zélande.

\begin{abstract}
Spatial variability of temperatures and climate change over the Marlborough wine-producing region of New Zealand

The objective of this research is to establish a methodology based on measurement (meteorological and agronomic) and spatial climate modeling suited to fine scale analysis, in order to define the current spatial climate variability and its evolution over vineyard regions within the context of climate change. The Marlborough region, the leading wine-producing region of New Zealand, is being investigated because of major local, regional and national concerns about the sustainability of wine production in this region. A first assessment of the temperature over a recent period, conducted using data from the national and two local meteorological networks, highlights strong spatial temperature variability at the regional scale, but mostly locally. Attention is paid to the occurrence of frosts, as well as the values of different bioclimatic indices used in viticulture. In addition, climate change projections were analyzed at fine scales for the medium term (2030-2049) and the end of the century (2080-2099), to provide the first indications about future local climate change across the Marlborough region.
\end{abstract}

Keywords: viticulture, climate change, local climate, Marlborough, New Zealand.

\section{Introduction}

D'après le dernier rapport du Groupe d'experts Intergouvernemental sur l'Evolution du Climat (GIEC), les différents modèles simulent une augmentation des températures (entre 2 et

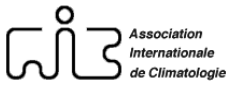


$6^{\circ} \mathrm{C}$ ) ainsi que de la fréquence et de l'intensité des phénomènes extrêmes (vagues de chaleur, ...) à l'horizon 2050-2100 (IPCC, 2013). Cette augmentation simulée varie suivant les régions du globe et selon les scénarios. Ces changements sont susceptibles d'être plus sensibles aux échelles fines, où l'influence des facteurs locaux engendre une variabilité spatiale du climat souvent plus importante que la variabilité temporelle liée au changement climatique d'échelle globale (Powell et al., 2011). De la même manière, les incertitudes quant aux évolutions possibles du climat augmentent avec la descente d'échelle spatiale, étant donné la multiplicité et la complexité grandissante des facteurs locaux influençant leur variabilité (Bonnefoy, 2013).

Au sein du monde viticole, ces éventuelles modifications du climat local sont source de nombreuses incertitudes, quant à la viabilité à long terme de la culture de certains cépages, notamment associés à des régions particulières, ou plus spécifiquement, à la pérennité de certains terroirs. La vigne est en effet extrêmement sensible aux variations climatiques (Quénol, 2012a), notamment aux températures qui influencent les différents stades phénologiques de la plante et conditionnent en grande partie la différentiation des terroirs viticoles exprimée à une échelle locale (Agenis-Nevers, 2006; Tondut et al., 2006 ; Van Leeuwen et al., 2008 ; Webb et al., 2008). Aussi, le changement climatique aura inévitablement des répercussions significatives sur l'activité viti-vinicole dans son ensemble (Bindi et al., 1996 ; Jones, 2007 ; Seguin, 2007). Par ailleurs, plusieurs études ont révélé les impacts d'ores et déjà observés du changement climatique sur la culture de certains cépages et sur les caractéristiques des terroirs viticoles, en Europe notamment (Schultz, 2000 ; Duchêne et Schneider, 2005 ; Jones et al., 2005a ; Seguin et Garcia De Cortazar Atauri, 2005 ; Chabin et al., 2007 ; Beltrando et Briche, 2010 ; Neethling et al., 2011 ; Briche et al., 2014). La hausse des températures serait, entre autres, à l'origine d'une modification des stades de développement du végétal et d'une modification des rendements (Bonnefoy et al., 2010 ; Madelin et al., 2010). Outre l'aspect phénologique, la qualité du vin produit, qui est souvent en relation avec les caractéristiques locales et micro-locales du milieu dans lequel il est produit, est elle aussi affectée par les modifications des paramètres climatiques, de manière hétérogène selon les régions du monde (Jones et al., 2005b ; Webb et al., 2008).

Dans ce contexte, les projets ANR-JC-TERVICLIM (2008-2012) et GICC-TERADCLIM (2011-2013) ${ }^{1}$, ont eu pour objectif la mise en place d'une méthodologie de mesures (météorologiques et agronomiques) et de modélisation spatiale du climat adaptée aux échelles fines, afin d'étudier la variabilité spatiale du climat et leurs évolutions à l'échelle de plusieurs régions viticoles. Cette meilleure compréhension du climat local, et de ses potentielles évolutions dans le contexte du changement climatique, devrait par la suite, permettre la mise en place de stratégies d'adaptation efficaces à destination des exploitants viticoles. Outre les grands vignobles français (Val de Loire, Champagne, Provence, Bourgogne et Bordeaux), le projet englobe des territoires viticoles de Roumanie, Espagne, Italie, Argentine, Chili, Bolivie, Uruguay, Afrique du Sud, Inde et Nouvelle-Zélande. L'envergure internationale du projet révèle cette prise de conscience généralisée quant aux potentiels impacts du changement climatique sur la culture de la vigne et la pérennité de certains terroirs viticoles ; cette adaptation est nécessaire à l'échelle globale, induite en partie par une concurrence sur le marché mondial qui ne cesse de grandir, et qui exacerbe les enjeux financiers liés à la production et à la commercialisation du vin (Rousset, 2004 ; Marre, 2004 ; Allaire et al., 2005 ; Quénol, 2012b).

Les recherches menées en Nouvelle-Zélande dans le cadre du projet "Development of advanced weather and climate modeling tools to help vineyard regions adapt to climate

\footnotetext{
${ }^{1}$ http://terviclim.in2p3.fr/spip.php?rubrique85
} 
change" (coordonné par Andrew Sturman), concernent la région de Marlborough qui, avec plus de 10000 hectares de vignes, constitue la première région productrice de vin du pays (Sluys, 2006 ; Powell et al., 2011). La région a connu à partir des années soixante-dix, un développement notable de sa filière viti-vinicole, du fait, entre autres, de climats locaux favorables et de techniques de production avancées, si bien que ses ventes sont aujourd'hui très compétitives sur les marchés internationaux, avec l'établissement d'un vin de qualité qui concurrence les meilleurs vins du monde (Moran, 2000). Aussi, la bonne compréhension de la variabilité du climat local, voire micro-local, et l'anticipation de ses potentiels changements à plus ou moins long terme, relève d'un enjeu majeur pour la filière viti-vinicole à l'échelle régionale et nationale.

La topographie de la région de Marlborough est particulièrement hétérogène. La complexité des facteurs influençant la variabilité locale à micro-locale des températures est potentiellement grande (Powell et al., 2011). Dans la continuité des travaux menés par Powell et al. (2011), l'analyse de la variabilité spatiale du climat constitue le premier objectif de cette recherche.

L'analyse à caractère exploratoire des récentes projections climatiques réalisées à échelle fine pour l'ensemble de la Nouvelle-Zélande pour le moyen terme et la fin du siècle, devrait permettre de mieux appréhender les potentielles modifications des caractéristiques climatiques locales, en lien avec la viticulture. Une évaluation des observations servant de référence aux projections est menée en amont, dans un double objectif d'identification des biais potentiels à associer aux projections futures, et d'alimentation des travaux en cours de modélisation à échelle fine au niveau du vignoble de Marlborough.

\section{Données et méthodes}

\subsection{Variabilité spatiale des températures}

A l'échelle de la région de Marlborough, le réseau météorologique national de la NIWA (National Institute of Water and Atmospheric Research ${ }^{2}$ ) se compose d'une trentaine de stations météorologiques enregistrant la température ${ }^{3}$, dont seize se trouvent dans les zones d'extension du vignoble. Parmi ces stations, très peu disposent de séries temporelles relativement longues (30 années). De plus, les dates des premières prises de mesures de la température sont très variables, réduisant ainsi considérablement les périodes avec des enregistrements communs entre les stations. Afin de disposer d'un réseau utile en termes de couverture temporelle et spatiale, les données issues de deux réseaux météorologiques locaux ont également été utilisées. Un réseau de stations météorologiques (Stu Powell ${ }^{4}$ et le Marlborough Wine Research Centre - dépendant de la compagnie scientifique nationale Plant \& Food Research ${ }^{5}$ ) a été implantées spécifiquement dans le vignoble de Marlborough, afin notamment de mesurer le risque gélif et, plus généralement, de comprendre les relations entre vignes et paramètres météorologiques.

Après analyse des périodes d'observations disponibles entre les stations des différents réseaux, une série temporelle de cinq ans (2008-2012) et 11 stations météorologiques ont finalement été retenues pour analyse (figure 1). La période d'observation a également été choisie en fonction des données manquantes, très limitées entre 2008 et 2012 pour les stations

\footnotetext{
${ }^{2}$ http://www.niwa.co.nz/

${ }^{3}$ Toutes les données d'observation de la NIWA sont disponibles en téléchargement depuis la base de données CliFlO (http://cliflo.niwa.co.nz/)

${ }^{4} \mathrm{http}: / /$ www.climateconsulting.co.nz/

${ }^{5} \mathrm{http}: / /$ www.plantandfood.co.nz/
} 
sélectionnées. Les séries sont en effet complètes à plus de $99 \%$, seuls quelques jours dispersés dans les séries étant manquants. Les stations de Wairau et Waihopai Valley font néanmoins exception, avec des lacunes de plus de 90 jours successifs. Les données de ces deux stations n'ont donc pas été utilisées sur l'ensemble de la période d'analyse avec les autres stations, mais leur utilisation reste tout à fait pertinente en dehors de ces périodes de données manquantes. Les séries de ces deux stations sont d'autant plus précieuses, qu'elles constituent la quasi-totalité des enregistrements de la température pour cette partie du vignoble.

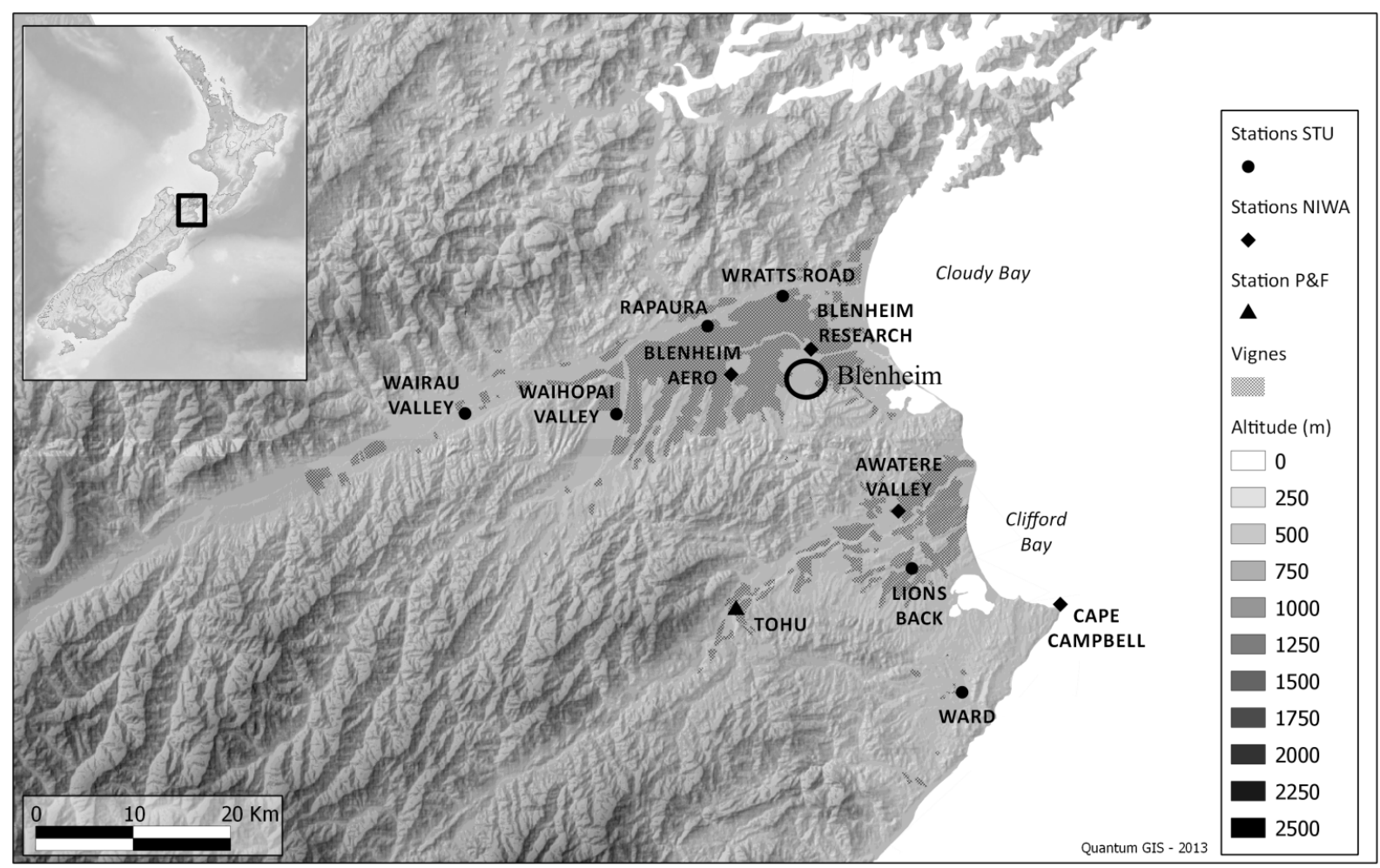

Figure 1 : Situation générale de la région de Marlborough et des 11 stations météorologiques utilisées pour l'analyse de la variabilité spatiale des températures à l'échelle du vignoble sur la période 2008-2012. General location of the Marlborough region and of the 11 weather stations used for the analysis of the temperature spatial variability over the vineyards between 2008 and 2012.

Les températures utilisées sont les moyennes journalières minimales, maximales et moyennes enregistrées à 1,5 mètres du sol. Les données journalières de vitesse et de direction du vent ont également fait l'objet d'analyses, celles-ci pouvant permettre d'expliquer une part de la variabilité thermique potentiellement observable à l'échelle du vignoble. Les données de température ont été analysées aussi bien en termes de moyenne que d'extrême, avec également une attention particulière portée à l'analyse de l'occurrence des gelées, notamment au printemps, saison durant laquelle la vigne est particulièrement sensible à cet aléa (Madelin, 2004 ; Madelin et Beltrando, 2005).

Par la suite, le calcul de plusieurs indices bioclimatiques a permis de définir les différents types de climats viticoles présents à l'échelle de la région de Marlborough. Ces indices, spécifiques à la viticulture, sont, entre autres, établis afin de caractériser les conditions optimales de culture de la vigne, en relation avec les températures principalement. L'indice des degrés jour de Winkler, l'indice héliothermique de Huglin et l'indice de fraîcheur des nuits sont les trois indices bioclimatiques calculés pour les différentes stations météorologiques sélectionnées (tableau 1). 


\begin{tabular}{|c|c|c|c|c|c|c|}
\hline \multirow{2}{*}{\multicolumn{3}{|c|}{$\begin{array}{c}\text { INDICE DE WINKLER } \\
\text { Valeur d'indice }\end{array}$}} & \multicolumn{2}{|c|}{ INDICE DE HUGLIN } & \multicolumn{2}{|c|}{ INDICE DE FRAICHEUR DES NUITS } \\
\hline & & & Type de climat & Valeur d'indice & \multirow{2}{*}{ Caractéristique } & \multirow{2}{*}{ Valeur d'indice } \\
\hline Région & ${ }^{\circ} \mathrm{C}$ & ${ }^{\circ} \mathrm{F}$ & \multirow{2}{*}{$\begin{array}{l}\text { Très frais } \\
\text { Frais }\end{array}$} & \multirow{2}{*}{$\begin{array}{c}\mathrm{IH} \leq 1500 \\
1500 \leq \mathrm{IH} \leq 1800\end{array}$} & & \\
\hline 1 & $<1371$ & $<2500$ & & & Nuits froides & $\mathrm{IF} \leq 12^{\circ} \mathrm{C}$ \\
\hline II & 1371 à 1649 & 2501 à 3000 & Tempéré & $1800 \leq \mathrm{IH} \leq 2100$ & Nuits très fraiches & $12^{\circ} \mathrm{C} \leq \mathrm{IF} \leq 14^{\circ} \mathrm{C}$ \\
\hline III & 1650 à 1926 & 3001 à 3500 & Tempéré chaud & $2100 \leq \mathrm{IH} \leq 2400$ & Nuits fraiches & $14^{\circ} \mathrm{C} \leq \mathrm{IF} \leq 16^{\circ} \mathrm{C}$ \\
\hline IV & 1927 à 2205 & 3501 à 4000 & Chaud & $2400 \leq \mathrm{IH} \leq 3000$ & Nuits tempérées & $16^{\circ} \mathrm{C} \leq \mathrm{IF} 18^{\circ} \mathrm{C}$ \\
\hline $\mathbf{v}$ & $>2205$ & $>4000$ & Très chaud & $\mathrm{IH} \geq 3000$ & Nuits chaudes & $\mathrm{IF} \geq 18^{\circ} \mathrm{C}$ \\
\hline
\end{tabular}

Tableau 1 : Valeurs relatives aux indices bioclimatiques de Huglin, de Winkler et de fraicheur des nuits, avec les caractéristiques des types de climats et des régions viticoles associées. Bioclimatic indices values and related wine climate types.

L'indice thermique de Winkler a été initialement établi pour caractériser les régions viticoles de Californie (Tonietto, 1999). Son calcul est simple et correspond à la somme des températures moyennes journalières supérieures à $10^{\circ} \mathrm{C}$ sur une période de 7 mois. Cette période s'étend du $1^{\mathrm{er}}$ avril au 30 octobre pour l'hémisphère Nord, et du $1^{\mathrm{er}}$ octobre au 30 avril pour l'hémisphère Sud.

L'indice de Huglin est un indice héliothermique qui prend en compte à la fois la température diurne et la durée du jour, une donnée tout à fait pertinente à considérer pour les vignobles situés aux latitudes moyennes comme le vignoble de Marlborough, situé entre 41 et $42^{\circ}$ de latitude. Cet indice fournit notamment des informations indirectes utiles sur la teneur en sucre du raisin (Tonietto, 1999). L'indice de Huglin se calcule selon la formule suivante :

$$
[(\mathrm{Tm}-10)+(\mathrm{Tx}-10) / 2] \times \mathrm{k}
$$

Où Tm est la température moyenne journalière, Tx la température maximale journalière et $\mathrm{K}$ le coefficient de longueur du jour variant entre 1,02 et 1,06 entre $40^{\circ}$ et $50^{\circ}$ de latitude.

La valeur de l'indice à une station donnée est ensuite obtenue par la somme des indices journaliers calculée sur une période de 6 mois, allant du $1^{\text {er }}$ avril au 30 septembre pour l'hémisphère Nord, et du $1^{\mathrm{er}}$ octobre au 31 mars pour l'hémisphère Sud.

L'indice de fraicheur des nuits correspond à la moyenne des températures minimales du mois qui précède les vendanges (Tonietto, 1999). Pour l'hémisphère Nord (Sud), il correspond donc à la moyenne des minimales du mois de septembre (mars). Cet indice est utile car il permet d'évaluer les arômes du raisin (Tonietto, 1999).

\subsection{Les projections à moyen et long terme à l'échelle de la région de Marlborough}

\subsubsection{L'élaboration des projections à échelle fine pour l'ensemble de la Nouvelle-Zélande}

Afin d'évaluer l'évolution future des températures et des précipitations à l'échelle de la Nouvelle-Zélande, les sorties issues de 12 modèles de circulation générale (MCG) ont été analysées. Ces modèles, utilisés pour élaborer les projections à l'échelle mondiale présentées dans le $4^{\text {ème }}$ rapport du Groupe d'experts Intergouvernemental sur l'Evolution du Climat (IPCC, 2007), ont fait l'objet d'une validation sur la période 1970-1999 pour la région du Pacifique Sud et la Nouvelle-Zélande (Mullan et Dean, 2009).

Les simulations ont été effectuées avec les scénarios A1B, A2 et B1 du SRES (IPCC, 2000), pour le moyen terme (2030-2049) et la fin du siècle (2080-2099), les évolutions étant relatives à la période de référence $1980-1999$, également utilisée dans le $4{ }^{\text {ème }}$ rapport de l'IPCC (2007). 
La résolution spatiale horizontale des modèles de circulation générale (de l'ordre de 200 à $300 \mathrm{~km}$ ) est trop grossière pour rendre compte de la complexité des changements à une échelle locale, ce qui est un problème récurrent dans le cadre des études d'impacts climatiques (Kim et al., 1984 ; Lamb, 1987 ; Cohen, 1990). L'élaboration de projections à échelle fine (quelques kilomètres) peut se faire par le biais d'une descente d'échelle statistique ou dynamique. La méthode de descente d'échelle dynamique consiste à utiliser les sorties d'un MCG pour faire tourner un nouveau modèle dynamique (c'est-à-dire capable de simuler la dynamique atmosphérique), sur une aire plus limitée ; les conditions aux limites du nouveau modèle étant contraintes par les sorties du MCG. Les évolutions proscrites par les scénarios d'émission des GES sont ensuite implantées dans le modèle à aire limitée, donnant ainsi des projections climatiques à des échelles spatiales avec une résolution de $10 \mathrm{~km}$ pour les modèles les plus performants. La seconde méthode consiste, dans un premier temps, à identifier les relations statistiques entre les évolutions des paramètres climatiques observées à large échelle (e.g. paramètres de circulation atmosphérique, champs de pression, températures moyennes), avec les évolutions des paramètres observées à une échelle régionale à locale (e.g. températures et précipitations), grâce aux réseaux d'observations et aux réanalyses climatiques. Ces relations, qui permettent de mettre en évidence l'influence des paramètres locaux (telle que la topographie), sont ensuite traduites sous forme de modèles de régression multiple, qui permettent de les formaliser (Mullan et al., 2002 ; Wilby et al., 2004 ; Madelin, 2004).

Privilégiée ici pour réaliser les projections à échelle fine pour l'ensemble de la NouvelleZélande, la descente d'échelle statistique a l'avantage d'être beaucoup moins coûteuse en temps de calcul. Elle est également un bon moyen de rendre compte des évolutions du climat à échelle fine, sans pour autant passer par l'établissement d'un modèle à aire limitée, qui reste relativement plus complexe à réaliser. Une phase préalable à cette descente d'échelle statistique a consisté en la création d'une grille régulière à partir de données d'observation à une échelle spatiale de $0,05^{\circ}$ (figure 2) pour l'ensemble de la Nouvelle-Zélande.

Figure 2 : Grille de résolution horizontale de $0,05^{\circ}$ (environ $5 \mathrm{~km}$ ) avec la topographie en arrière plan au niveau de la région de Marlborough. Spatial grid of $0.05^{\circ}$ over the Marlborough region overlaid on the topography.

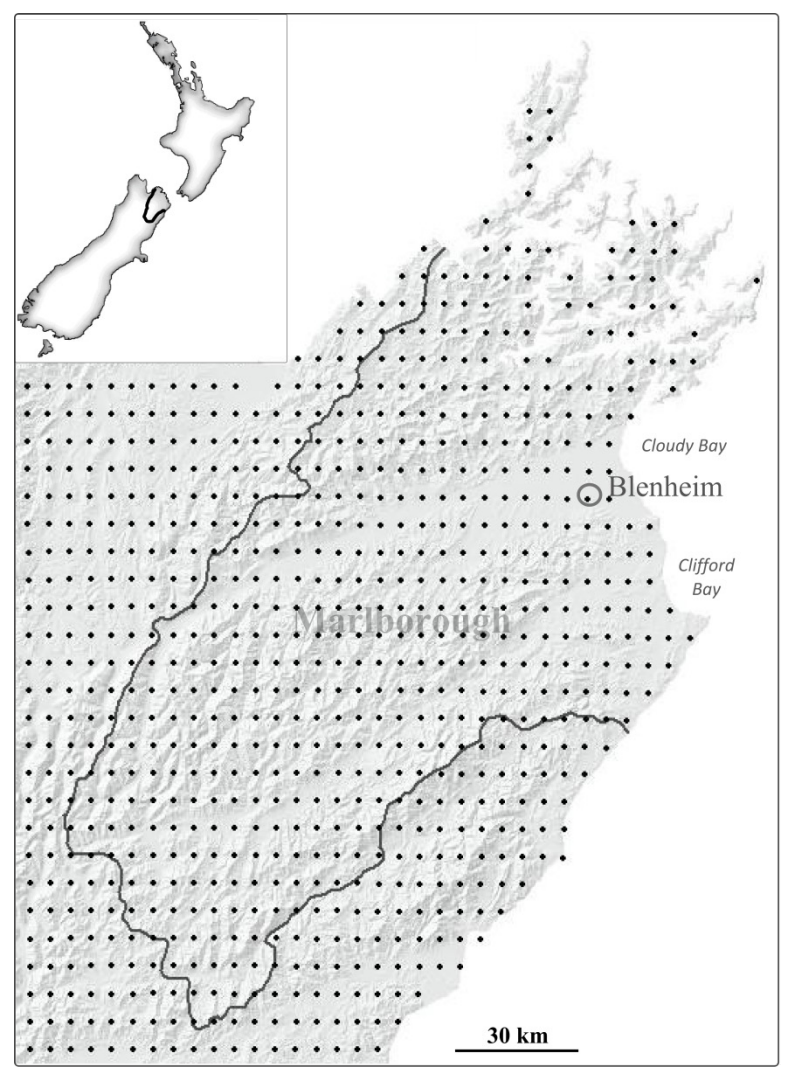


Ces champs climatique, qui couvrent actuellement une période de 40 ans entre 1972 et 2012, sont, entre autres, relatifs aux températures minimales et maximales, ainsi qu'aux précipitations journalières ; ils ont été obtenus à partir de l'interpolation spatiale des données d'observation issues du réseau de stations météorologiques de la NIWA (Tait et al., 2006), et sont disponibles dans leur intégralité via la base de données $\mathrm{CliFlO}^{6}$.

A partir de ces champs de température et de précipitation calculés sur une grille de $0,05^{\circ}$, et avec les réanalyses issues du NCEP (National Centers for Environnemental Prediction ${ }^{7}$ ) rendant compte de l'évolution de paramètres atmosphériques à large échelle ${ }^{8}$, les modèles de régression liant variables observées à une échelle locale et synoptique ont pu être établis, la méthode utilisée étant similaire à celle décrite par Mullan et al. (2002).

Les sorties des 12 modèles de circulation générale pour les deux futures périodes (20302049 et 2080-2099) et les trois scénarios d'émission (A1B, A2 et B1), ont été intégrés dans les modèles de régression pour obtenir des projections climatiques à la résolution spatiale horizontale de $0,05^{\circ}$ pour l'ensemble du pays. Cette descente d'échelle a été effectuée pour les températures et précipitations moyennes mensuelles, sachant que la descente d'échelle des températures minimales et maximales devrait être réalisée prochainement en 2014.

\subsubsection{Le traitement des données issues de la descente d'échelle statistique des températures pour les deux futures périodes}

La base de données des projections à échelle fine des températures moyennes mensuelles se caractérise par l'écart thermique entre la normale (1980-1999) et les périodes futures (20302049 et 2080-2100). Afin, entre autres, de rendre compte de l'évolution du nombre de jours de gel (notamment au printemps), et des indices bioclimatiques faisant l'objet d'une analyse sur la période récente (2008-2012), ces écarts thermiques entre les deux périodes ont été appliqués aux températures minimales, maximales et moyennes journalières. Il s'agit ici d'un travail exploratoire, qu'il serait intéressant de compléter par la suite avec les potentielles nouvelles données relatives aux projections (notamment les données issues de la descente d'échelle des températures minimales et maximales). Une moyenne des douze modèles de climat a été privilégiée, afin notamment de réduire l'incertitude inhérente à chaque simulation et/ou modèle.

\subsubsection{Une évaluation des champs climatiques élaborés sur la grille de résolution horizontale $0,05^{\circ}$}

La méthode utilisée pour évaluer ces données consiste à comparer les données d'observation réelles d'une station avec les données d'observation virtuelles correspondant au point de grille le plus proche. A partir des données journalières de température, la qualité de la reproduction des valeurs moyennes, minimales, maximales, ainsi que des extrêmes (notamment les minimales printanières) et des valeurs d'indices bioclimatiques (Huglin, Winkler et fraicheur des nuits) a été évaluée.

Dans un premier temps, l'évaluation a porté sur la période de référence 1980-1999. Pour ce faire, les données de température et de précipitation de 7 points de grille ont été comparées aux données issues des 9 stations NIWA les plus proches (figure 3). Sur cette période, les seules données d'observation directes existantes sont celles de la NIWA, données qui ont servi à l'interpolation. Dans un deuxième temps, afin d'approfondir cette évaluation, les

\footnotetext{
${ }^{6} \mathrm{http}: / /$ cliflo.niwa.co.nz/

7 http://www.ncep.noaa.gov/

${ }^{8}$ Les paramètres de large échelle utilisés pour l'élaboration des modèles de régression sont les températures et précipitations moyennes, l'indice Trenberth Z1 (permettant de rendre compte de l'intensité du flux zonal d'ouest), ainsi que l'indice M1 (permettant de rendre compte de l'intensité du flux de secteur sud).
} 
données de température de 6 points de grille ont été comparées avec les observations de 6 stations du réseau de Stu Powell sur la période 2006-2012 (figure 3), permettant ainsi de tester la qualité de l'interpolation avec un réseau de données n'ayant pas servi à l'interpolation.
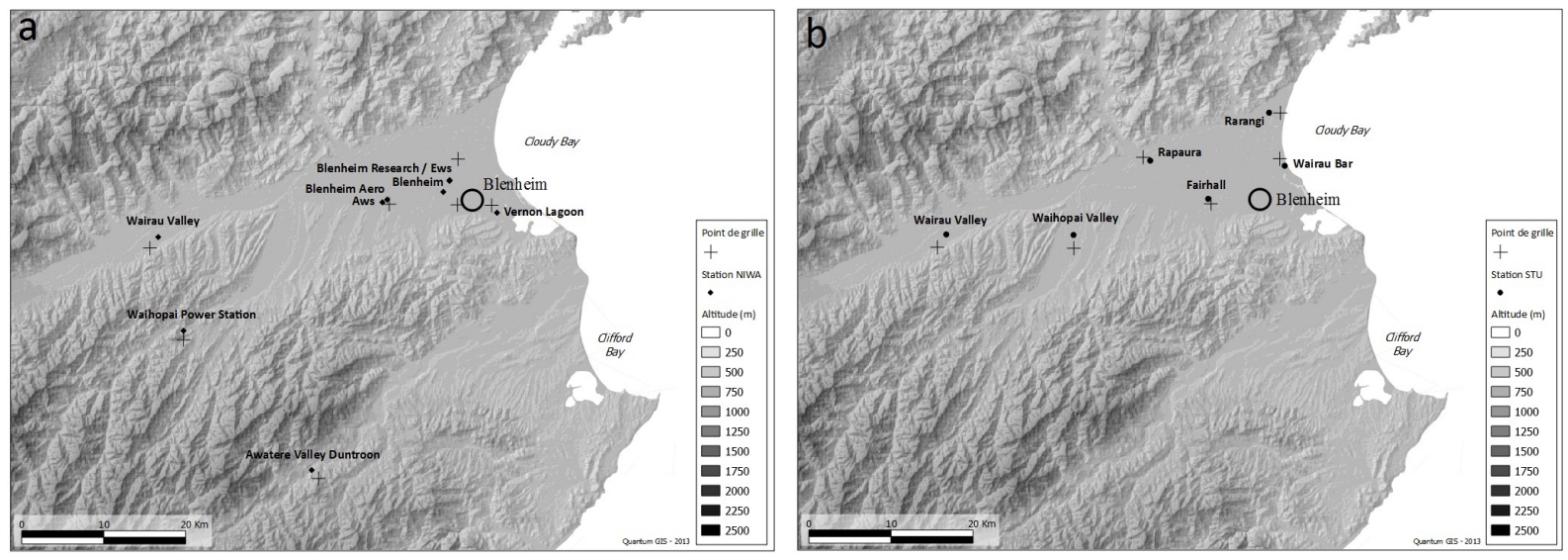

Figure 3: Situation des stations du réseau de la NIWA (a) et du réseau de Stu Powell (b) utilisées pour l'évaluation des données recalculées par interpolation sur la période 1980-1999 (évaluation avec le réseau de la NIWA) et 2006-2012 (évaluation avec le réseau de Stu Powell). Les données issues de ces différentes stations ont été comparées avec les données du point de grille (données virtuelles) le plus proche. Location of the NIWA stations (a) and Stu Powell stations (b) used for the evaluation of the virtual observation data for the period 1980-1999 (evaluation using NIWA stations) and 2006-2012 (evaluation using Stu Powell stations). The real observation data have been compared with the virtual observations calculated at the nearest grid point.

\section{Résultats}

\subsection{Variabilité spatiale des températures sur la période 2008-2012}

\subsubsection{Une forte variabilité à l'échelle de la région d'étude}

Une première analyse des températures au niveau des 11 stations météorologiques (ayant des données de 2008 à 2012), permet de mettre en évidence une influence maritime à l'échelle régionale. D'une manière générale, l'amplitude thermique moyenne mensuelle enregistrée au niveau des stations tend à augmenter avec la distance à la mer. Les stations de Waihopai Valley, Wairau Valley et Tohu sont les plus éloignées des côtes, et enregistrent les amplitudes thermiques moyennes mensuelles les plus importantes sur la période 2008-2012, entre 13 et $14^{\circ} \mathrm{C}$, pour des valeurs minimales de l'ordre de $5^{\circ} \mathrm{C}$ et des maximales autours de $18,5^{\circ} \mathrm{C}$. A l'inverse, la station de Cape Campbell, située en bord de mer, se distingue particulièrement des autres. L'amplitude thermique moyenne mensuelle y est en effet relativement réduite (figure 4).

Figure 4 : Amplitudes thermiques moyennes mensuelles de 4 stations NIWA analysées sur la période 2008-2012. Monthly mean temperature range of the 4 NIWA weather stations analysed over the 2008-2012 period.

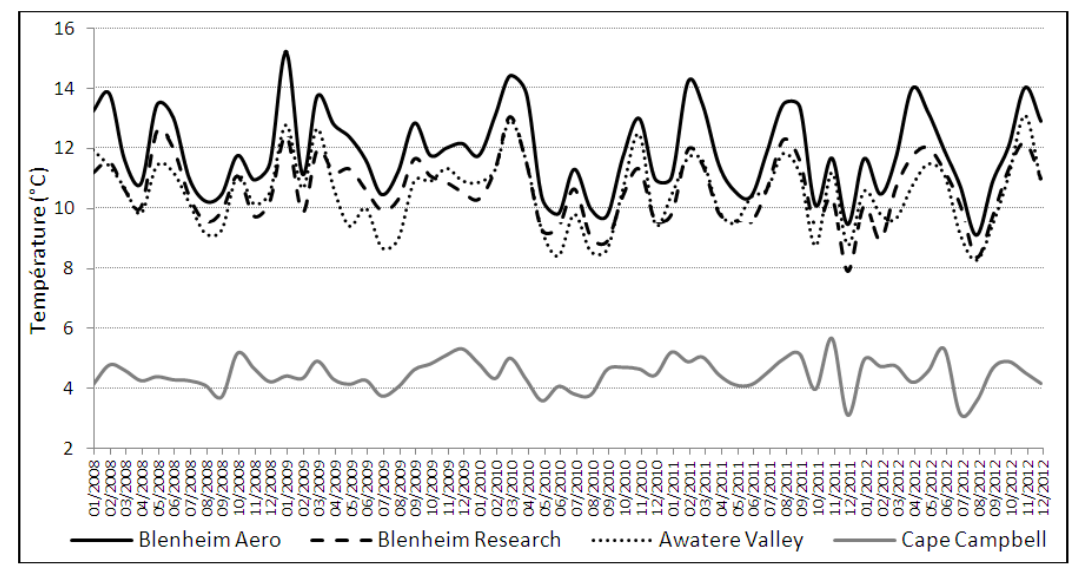


Outre l'inertie thermique, la variabilité spatiale des températures due à l'influence maritime peut également être mise en évidence à l'échelle régionale au travers de l'analyse du nombre de jours de gel $\left(\mathrm{Tn}<=00^{\circ} \mathrm{C}\right)$ et de fortes gelées $\left(\mathrm{Tn}<=-2^{\circ} \mathrm{C}\right)$, particulièrement importants à considérer pour leurs impacts connus sur les cépages (Madelin, 2004). A l'échelle de la région de Marlborough, l'occurrence des gelées annuelles est en effet tributaire de l'éloignement à la mer à près de $50 \%$. Le nombre de jours durant lesquels la température atteint ou descend en dessous de $-2^{\circ} \mathrm{C}$ est également dépendant de la distance à l'océan selon un coefficient de détermination $\left(\mathrm{R}^{2}\right)$ de 0,45 (figure 5).

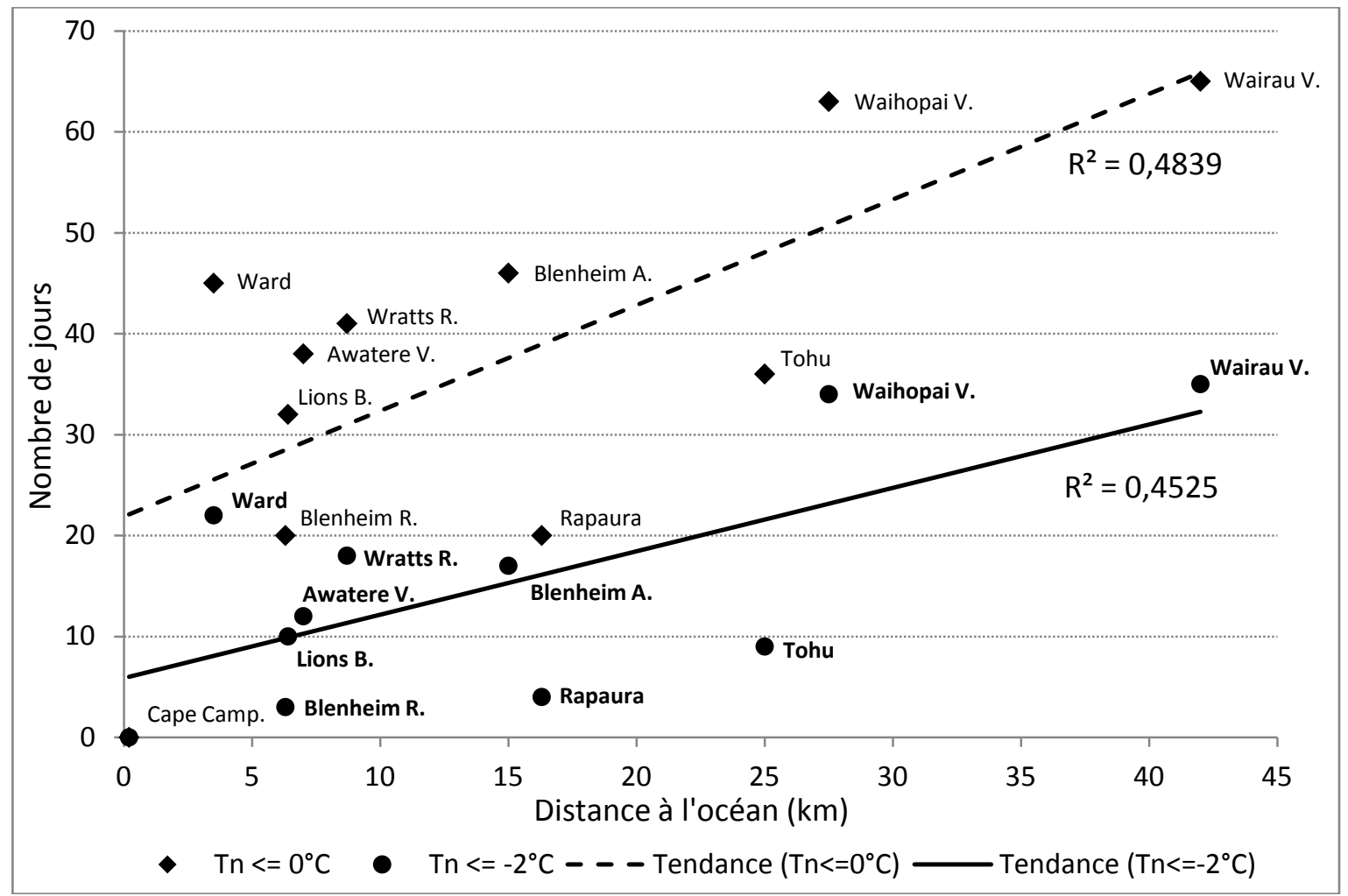

Figure 5 : Graphique bivarié montrant la relation linéaire entre d'une part la distance à la mer (en km ; axe des abscisses) et d'autres part (axes des ordonnées) le nombre de jours de gel $\left(\mathrm{Tn}<=0^{\circ} \mathrm{C}\right)$ et de forte gelée ( $\operatorname{Tn}<=$ $2^{\circ} \mathrm{C}$ ) moyen annuel sur la période 2008-2012 pour les 11 stations analysées. Annual mean of the number of days of frost $\left(\mathrm{Tn}<=0^{\circ} \mathrm{C}\right)$ and heavy frost $\left(\mathrm{Tn}<=-2^{\circ} \mathrm{C}\right)$ at the 11 weather stations analysed over the 2008-2012 period in relationship with their distance from the sea.

Les résultats montrent que l'influence maritime ne constitue pas le seul facteur explicatif potentiel de la variabilité régionale de l'occurrence des gelées et fortes gelées. La variabilité du nombre de jours de gel moyen annuel est plus importante au niveau de la vallée nord par rapport à celle au sud, avec un nombre de jours de gel inférieur à 10 en bord de mer et supérieur à 60 en zone amont, pour les stations de Waihopai et Wairau Valley (figure $6^{9}$ ). Dans la vallée sud, le nombre de jours de gel moyen annuel atteint son maximum à la station de Ward avec 45 occurrences. La station de Tohu enregistre entre 35 et 40 jours de gel par an. Le contraste est donc visible entre les parties amont des deux principales vallées de la région, l'une étant concernée par une plus grande fréquence de gelée que l'autre. Cette différence se retrouve au printemps, période durant laquelle seulement 3 occurrences de gelée sont

\footnotetext{
${ }^{9}$ Sur le plan géostatistique, la figure 6 montre de forts « effet pépite » liés aux stations. La cartographie obtenue est fortement dépendante du nombre de stations et de leur répartition. Ce type de document cartographique est avant tout indicatif et en aucun cas utilisable pour des calculs de cumuls hydrologiques ou climatiques liés aux superficies.
} 
enregistrées au niveau de la station de Tohu, contre 10 et 8 enregistrées respectivement au niveau des stations de Waihopai et Wairau Valley (tableau 2).

Tableau 2: Occurrences moyennes de jours de gel $\left(\mathrm{Tn}<=0^{\circ} \mathrm{C}\right)$ au printemps (SON) sur la période 2008-2012 pour les 11 stations analysées. Mean spring frost occurrence at the 11 weather stations analysed for the period 2008-2012.

\begin{tabular}{|cc|}
\hline Station & Jour de gel printanier \\
\hline Wairau & 10 \\
\hline Waihopai & 8 \\
\hline Blenheim Aero AWS (4326) & 6 \\
\hline Awatere Valley (18468) & 6 \\
\hline Ward & 4 \\
\hline Wratts Road & 3 \\
\hline Tohu & 3 \\
\hline Lions Back & 2 \\
\hline Blenheim Research (12430) & 1 \\
\hline Rapaura & 1 \\
\hline Cape Campbell (4424) & 0 \\
\hline
\end{tabular}

La topographie est le principal facteur expliquant cette forte variabilité spatiale, les vallées de Waihopai et Wairau étant plus fréquemment en situation d'abri vis-à-vis des vents synoptiques, favorisant ainsi les situations de vents calmes et la stagnation d'air froid au niveau de la couche atmosphérique de surface. Le facteur topographique peut également expliquer la moins grande occurrence de gelée enregistrée au niveau de la station de Rapaura par rapport aux stations de Wratts Road et Blenheim Aero. En effet, directement au nord de la station, se trouve un couloir topographique (figure 6). Orienté selon un axe nord/sud et ouvert sur la mer plus au nord, ce couloir favorise localement la circulation d'un air plus doux relativement à la masse d'air froid potentiellement en place au niveau de la vallée principale nord.

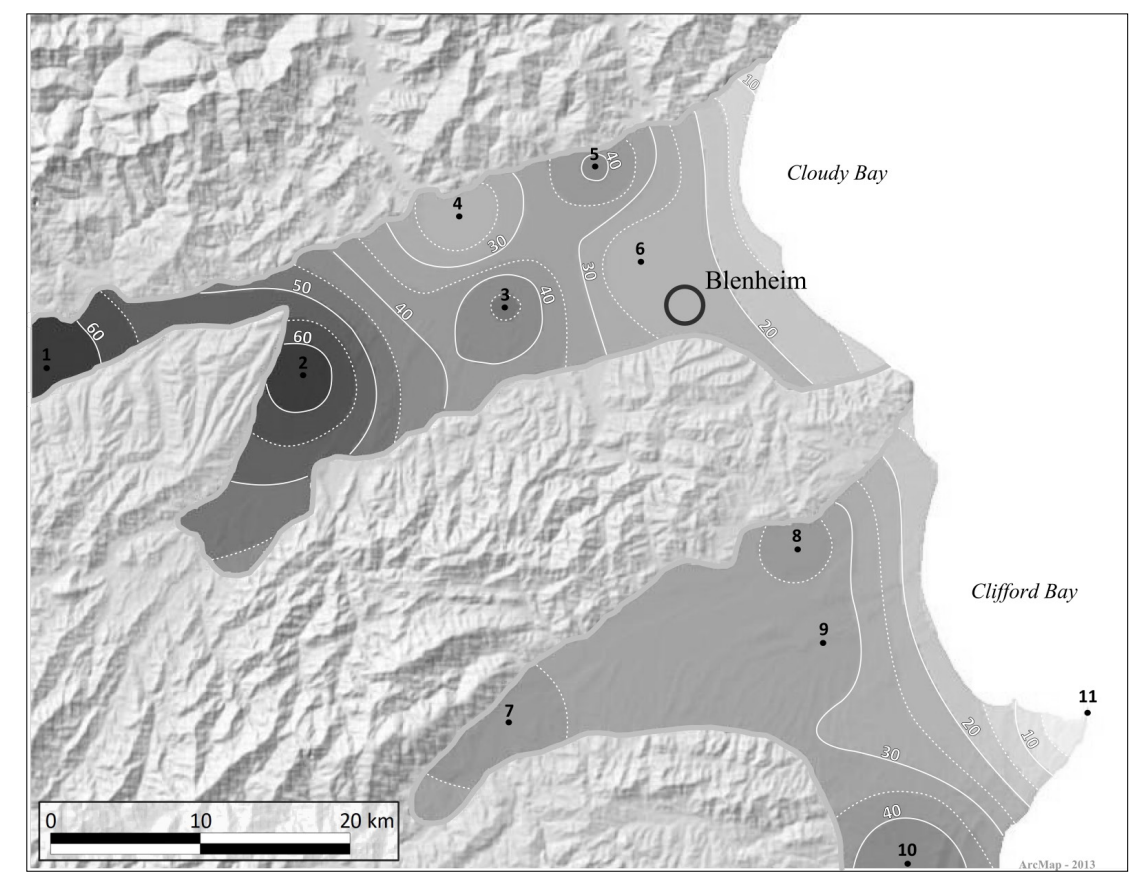

Figure 6: Nombre de jours de gel (intervalle des isothermes : 5 jours) moyen annuel calculé sur la période 2008-2012 à l'échelle de la région de Marlborough (réalisation sous ArcMap ${ }^{\circledR}$ selon une méthode d'interpolation IDW réalisée à partir des données calculées au niveau des 11 stations météorologiques analysées : 1 Wairau Valley ; 2 Waihopai Valley ; 3 Blenheim Aero ; 4 Rapaura ; 5 Wratts Road ; 6 Blenheim Research ; 7 Tohu ; 8 Awatere Valley ; 9 Lions Back ; 10 Ward ; 11 Cape Campbell). Mean annual number of days of frost (isotherm interval = 5 days) over the 2008-2012 period for the Marlborough region (Map built using ArcMap and an IDW interpolation method - 1 Wairau Valley; 2 Waihopai Valley; 3 Blenheim Aero; 4 Rapaura; 5 Wratts Road; 6 Blenheim Research; 7 Tohu; 8 Awatere Valley; 9 Lions Back; 10 Ward; 11 Cape Campbell). 


\subsubsection{Une forte variabilité spatiale des températures engendrée par des facteurs locaux}

La topographie complexe de la région de Marlborough engendre une forte variabilité des températures à une échelle locale. Les différences d'exposition aux vents synoptiques, ainsi que les modes complexes d'écoulement de l'air froid, notamment lors de situations radiatives de ciel clair et de vent calme (Beltrando, 1998), sont autant de paramètres dépendants de la topographie et qui peuvent engendrer de très fortes disparités locales de la température. Le travail mené par Powell et al. (2011), par le biais de l'analyse des données issues d'un réseau de capteurs répartis à l'échelle du vignoble de la région de Marlborough, a permis de mettre en évidence cette forte variabilité locale des températures. En considérant les données journalières de températures des différentes stations analysées ici sur la période 2008-2012, cette variabilité thermique locale est explicite, notamment pour les températures minimales. L'exemple est donné pour les stations de Rapaura et Wratts Road, distantes de $8 \mathrm{~km}$ et où un écart de plus de $13,5^{\circ} \mathrm{C}$ est enregistré au cours du mois de mars 2010 (figure 7), le facteur topographique expliquant de telles amplitudes.

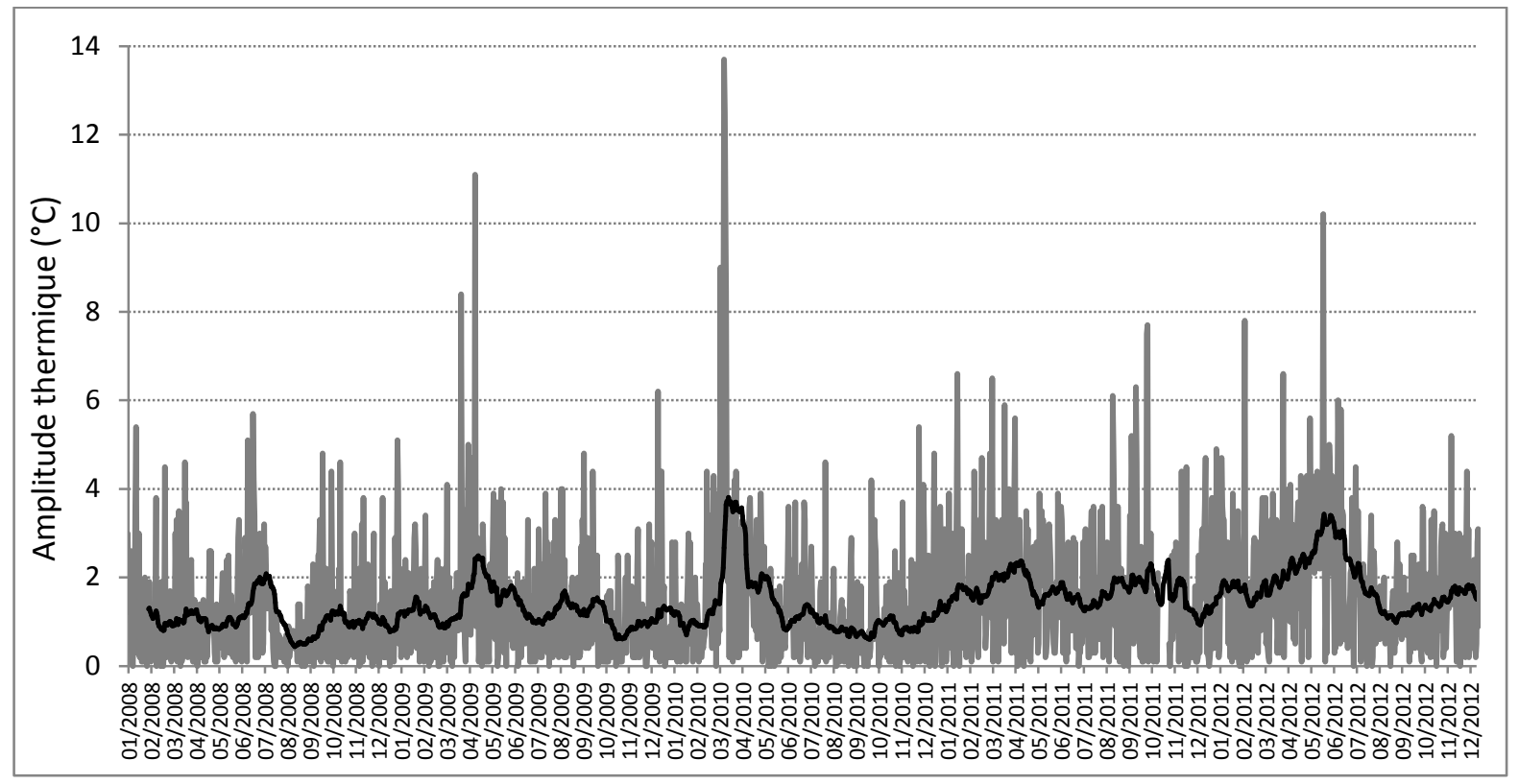

Figure 7 : Amplitude thermique journalière (courbe grise) calculée entre les températures minimales journalières des stations de Rapaura et Wratts Road sur la période 2008-2012, et moyenne mobile sur une période de 30 jours (courbe noire). Grey line: daily temperature difference between minimal temperatures of Rapaura and Wratts Road over the 2008-2012 period. Black line: moving average over 30 days.

L'analyse des extrêmes froids au niveau des stations de Lions Back et Ward, distantes de $13 \mathrm{~km}$, montre d'avantage l'importance des conditions topographiques locales sur les températures. Sur la période 2008-2012, la station de Ward, située à 3,5 km de la côte, enregistre une plus grande fréquence d'extrêmes froids par rapport à la station de Lions Back, pourtant située $3 \mathrm{~km}$ plus à l'intérieur des terres (figure 8). Aussi, l'influence océanique mise en évidence précédemment à l'échelle régionale, ne peut dans ce cas, pas expliquer la plus grande fréquence d'extrêmes froids enregistrés à Ward. Les caractéristiques topographiques de ces stations peuvent en revanche permettre d'expliquer une part de cette variabilité. En effet, la station de Ward, contrairement à Lions Back, ne se trouve pas dans l'axe de la vallée principale d'Awatere, et se trouve davantage en situation d'abri. La vitesse du vent journalier au niveau de Ward est en effet nettement inférieure à celle enregistrée à Lions Back (figure 9). Aussi, les situations de gel radiatif sont plus fréquentes au niveau de la station de Ward, ce qui explique, en partie, la plus grande fréquence d'extrêmes froids observés au niveau de cette station par rapport à Lions Back.

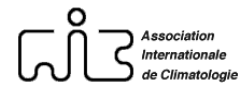


Figure 8 : Fréquences thermiques des températures inférieures à $0^{\circ} \mathrm{C}$ aux stations de Lions Back et Ward sur la période moyenne 2008-2012. Frequency of the temperatures below $0^{\circ} \mathrm{C}$ at Lions Back and Ward weather stations over the 20082012 period.
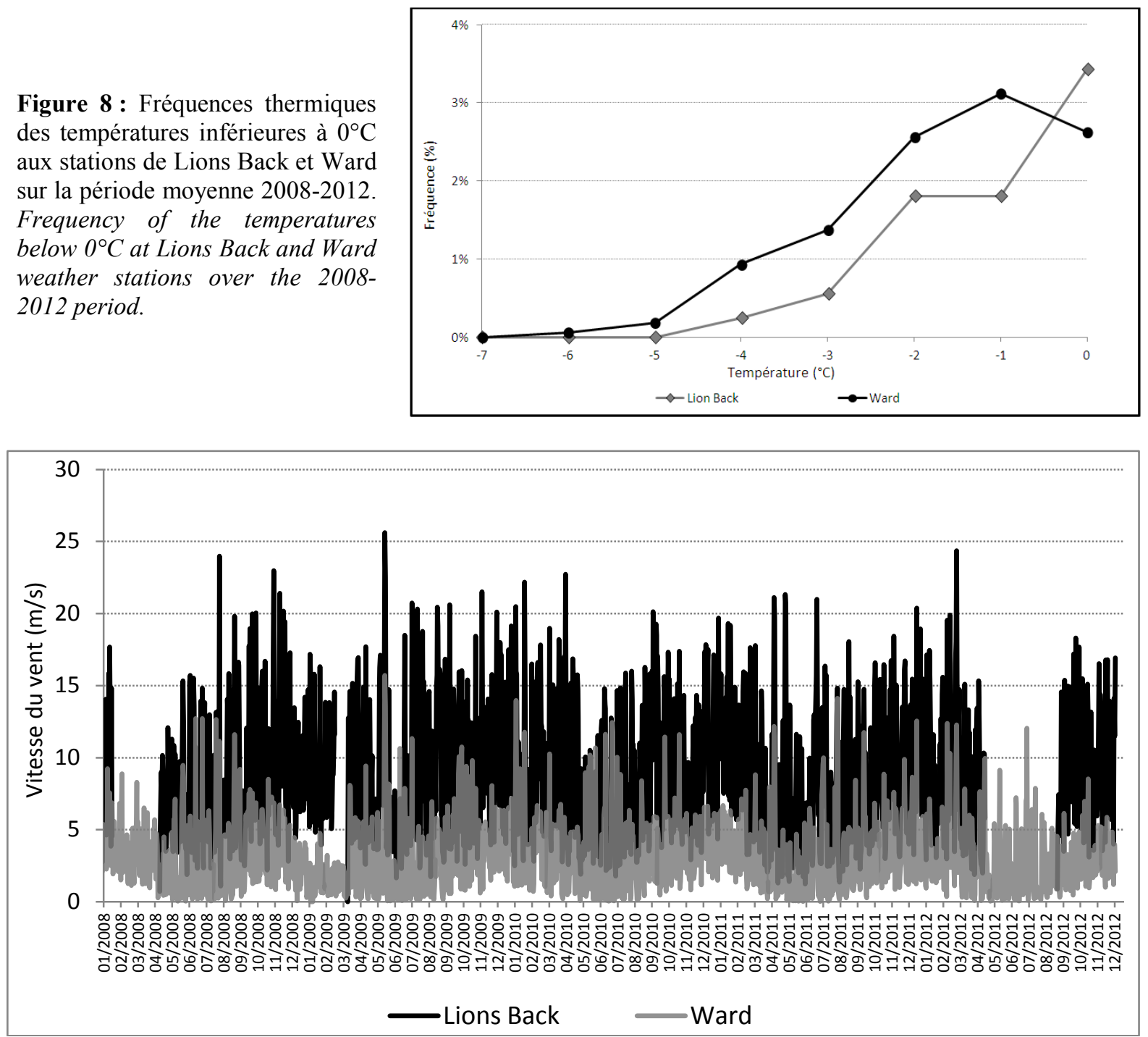

Figure 9 : Vitesse du vent journalier entre 2008 et 2012 enregistrée aux stations de Lions Back et Ward. Daily wind speed between 2008 and 2012 recorded at Lions Back and Ward.

\subsubsection{Impact sur les indices bioclimatiques}

D'une manière générale, les types de climat viticole sont relativement frais. Selon l'indice de Huglin, la région est caractérisée entre 2008 et 2011 par un climat allant d'un type très frais (indice inférieur à 1500) en s'appuyant sur les stations de Cape Campbell et Ward, à un type frais (indice compris entre 1500 et 1800) à l'échelle des autres stations (mis à part la station de Lions Back qui enregistre entre 2008 et 2009 un indice inférieur à 1500). Entre 2011 et 2012, l'ensemble des stations analysées enregistre des indices de Huglin inférieurs à 1500 (figure 10).

Les valeurs d'indice de fraicheur des nuits mettent également en évidence deux types de climats viticoles à l'échelle de la région. L'un est caractérisé par des nuits très fraiches (indice compris entre 12 et $14^{\circ} \mathrm{C}$ ) et s'observe sur toute la période d'analyse au niveau de la station de Cape Campbell, alors que l'autre est typiques de nuits froides (indice inférieur à $12^{\circ} \mathrm{C}$ ) et s'observe au niveau des 10 autres stations, excepté à Lions Back où est obtenu un indice supérieur à $12^{\circ} \mathrm{C}$ en 2008 (figure 11). Les valeurs d'indice de Winkler ne révèlent en revanche pas de différenciation de type de climat viticole à l'échelle de Marlborough, et seule la région I (indice inférieur à 1371) est mise en évidence entre 2008 et 2012. 


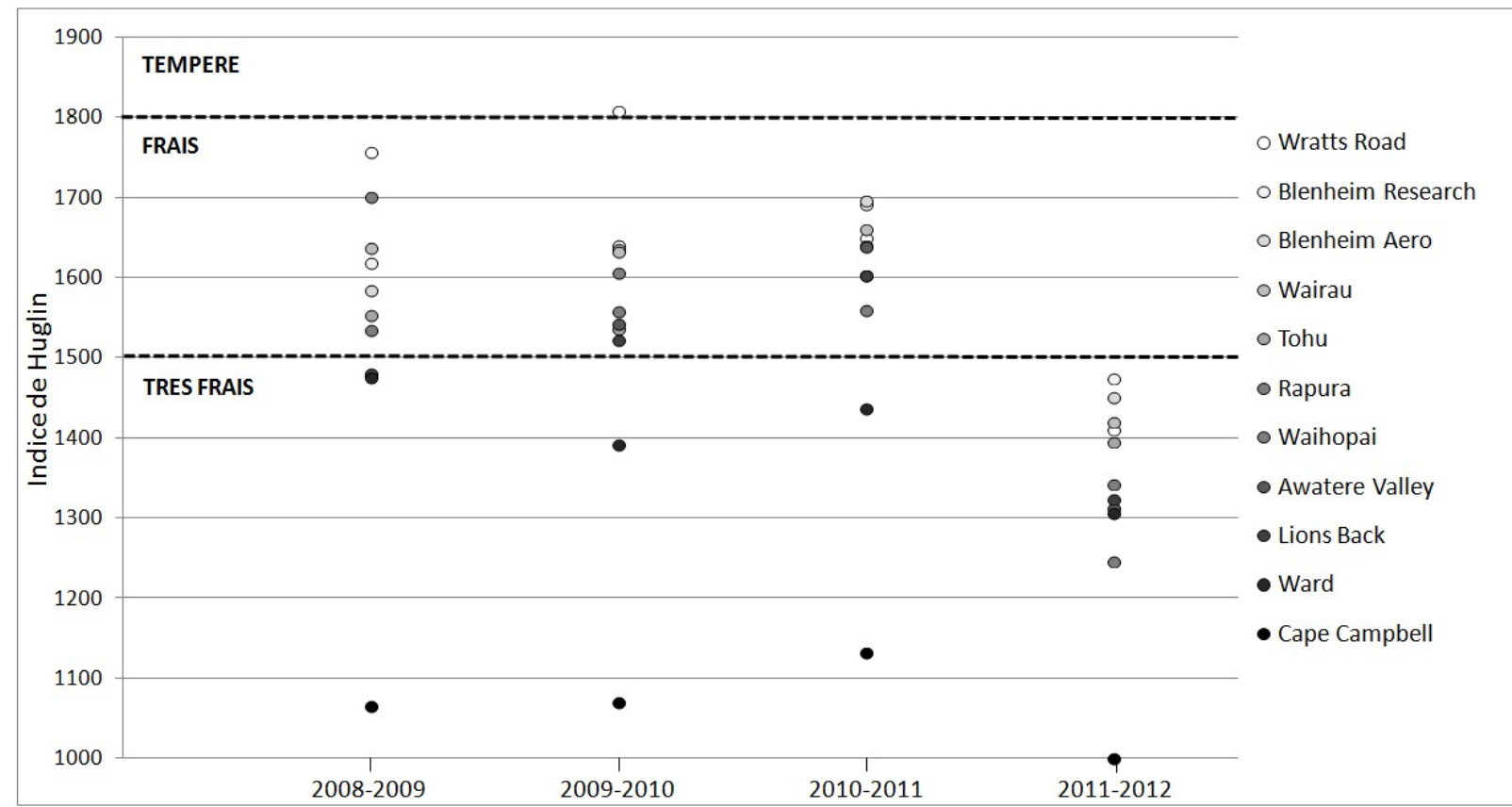

Figure 10 : Indices d'Huglin des 11 stations analysées sur la période 2008-2012 et climats viticoles associés. Le dégradé de gris dans la légende rend compte de la hiérarchisation associée à la moyenne des valeurs sur la période : e.g. Wratts Road a la moyenne des valeurs d'indice de Huglin la plus élevée sur la période 2008-2012, Cape Campbell la plus basse. Huglin index values of the 11 weather stations analysed over the 2008-2012 period and related viticultural climates. The average index value was calculated for the whole period 2008-2012 for each station, the grey gradient in the legend reflects the hierarchy associated with this average: e.g. Wratts Road got the highest Huglin index average over the 2008-2012 period, Cape Campbell the lowest.

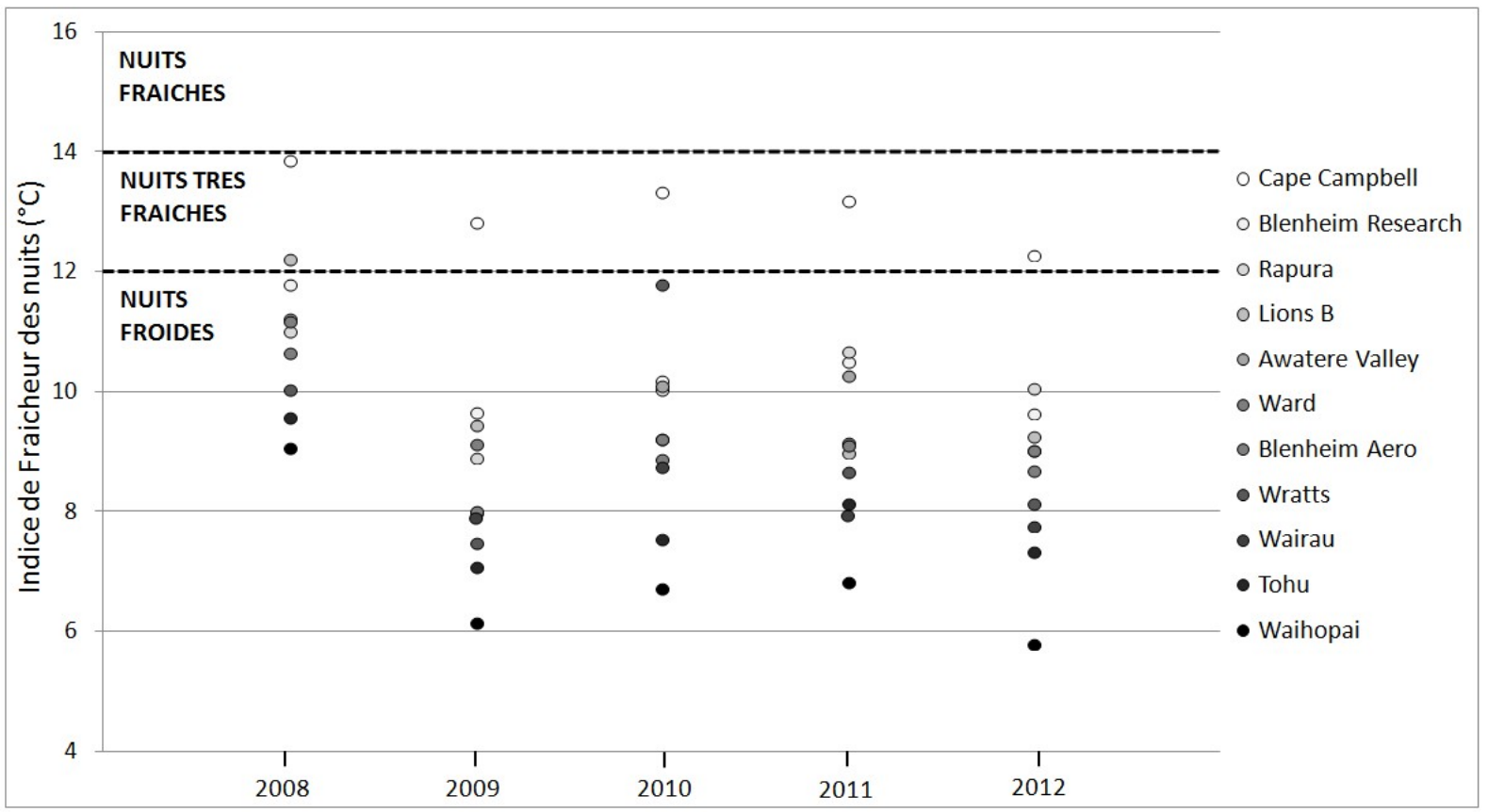

Figure 11 : Indices de fraicheur des nuits des 11 stations analysées sur la période 2008-2012. Le dégradé de gris dans la légende rend compte de la hiérarchisation associée à la moyenne des valeurs sur la période : e.g. Cape Campbell a la moyenne des valeurs d'indice de fraicheur des nuits la plus élevée sur la période 2008-2012, Waihopai la plus basse. Cold night index values of the 11 weather stations analyzed over the 2008-2012 period. The average index value was calculated for the whole period 2008-2012 for each station, the grey gradient in the legend reflects the hierarchy associated with this average: e.g. Cape Campbell had the highest cold nights index average over the period 2008-2012, Waihopai the lowest. 


\subsection{L'évolution climatique observée et simulée à l'échelle de la région de Marlborough}

\subsubsection{L'augmentation prévue des températures}

A l'échelle planétaire, l'augmentation de la température moyenne annuelle pour l'horizon 2030-2049, relativement à la période de référence 1980-1999, devrait être comprise entre 1 et $1,2^{\circ} \mathrm{C}$, respectivement pour le scénario le plus optimiste (B1) et celui le plus pessimiste (A2) du GIEC. D'ici la fin du siècle (2080-2099), l'augmentation devrait être comprise entre 1,5 et $3,3^{\circ} \mathrm{C}$, respectivement pour les scénarios B1 et A2 du GIEC (IPCC, 2007).

A l'échelle de la Nouvelle-Zélande, une augmentation des températures de $0,9^{\circ} \mathrm{C}$ a d'ores et déjà été enregistrée sur les 100 dernières années (Mullan et al., 2008). Suivant cette tendance, l'augmentation de la température moyenne annuelle relativement à la période de référence 1980-1999, devrait être comprise entre 0,7 et $0,9^{\circ} \mathrm{C}$ à moyen terme (2030-2049), et entre 1,3 et $2,5^{\circ} \mathrm{C}$ pour la fin du siècle (2080-2099), respectivement pour les scénarios B1 et A2 du GIEC (Reisinger et al., 2010); une augmentation à l'échelle nationale qui devrait donc être moins importante que celle prévue à l'échelle globale.

L'analyse des projections thermiques pour les deux futures périodes à l'échelle de la région de Marlborough rend compte d'une augmentation quasi similaire à celle qui est prévue à l'échelle du pays. En effet, en considérant uniquement les points de grille couvrant la région, la hausse de la température moyenne annuelle devrait être comprise entre 0,7 et $0,9^{\circ} \mathrm{C}$ à moyen terme, et entre 1,3 et $2,6^{\circ} \mathrm{C}$ pour la fin du siècle, respectivement pour les scénarios $\mathrm{B} 1$ et A2 du GIEC (figure 12).

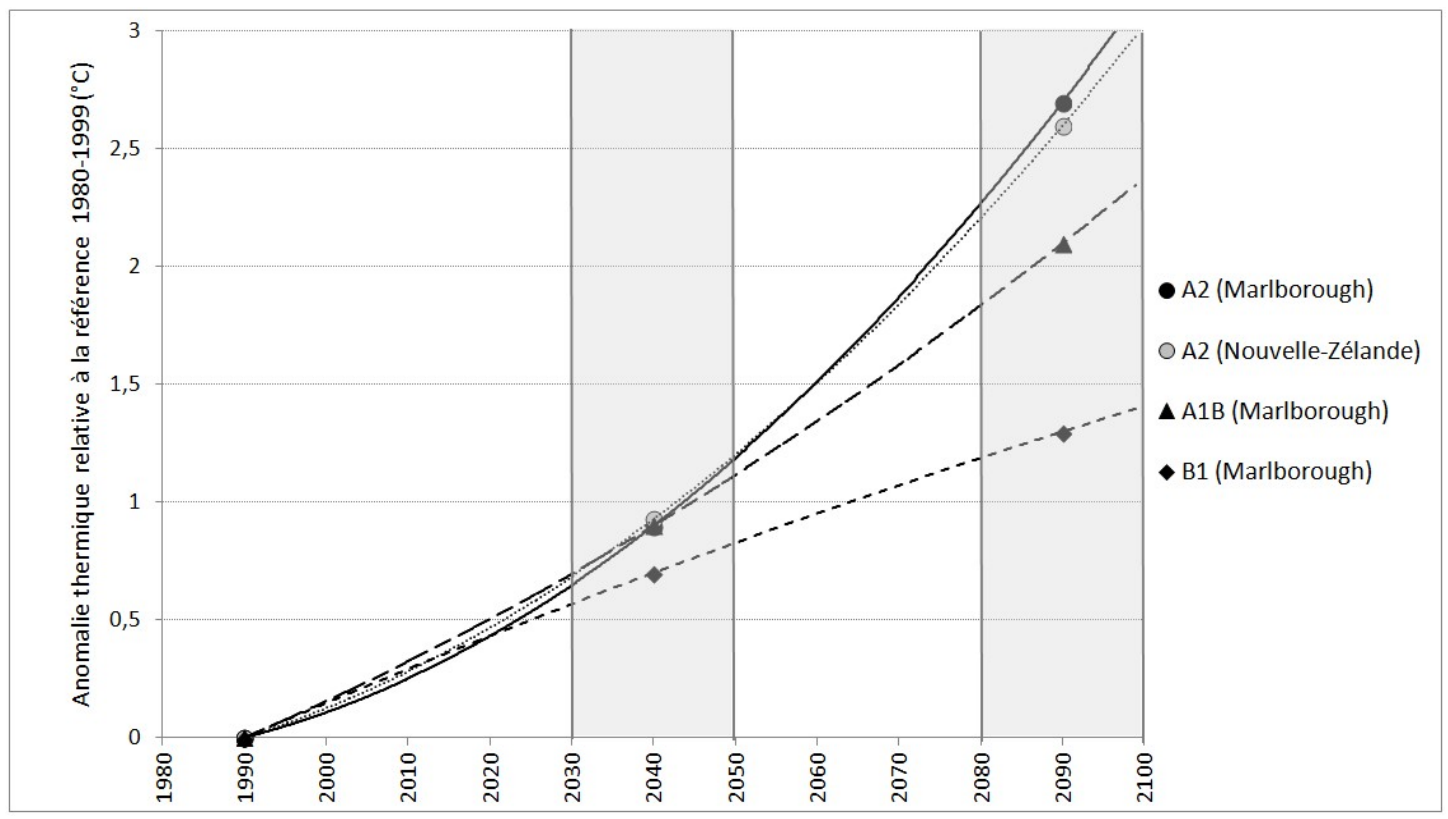

Figure 12 : Anomalies thermiques moyennes annuelles prévues pour les périodes 2030-2049 et 2080-2099 par rapport à la référence 1980-1999 et selon différents scénarios du GIEC. Les anomalies sont relatives à la moyenne des températures calculée sur une période de 20 ans; seuls les points correspondent à une information effective, les courbes sont des tendances polynomiales qui permettent simplement de mieux visualiser les hausses sur l'ensemble de la période entre 1980 et 2100. Pour l'ensemble de la Nouvelle-Zélande, seule l'augmentation selon le scénario A2 est indiquée sur le graphique, étant donné que les augmentations prévues pour les scénarios A1B et B1 sont similaires à celles prévues pour la région de Marlborough. Mean Annual thermal anomalies expected for 2030-2049 and 2080-2099 in relation to the reference period 1980-1999 and different IPCC scenarios. Anomalies are relative to the average temperature over a period of 20 years, and only the points correspond to effective information, while the curves are polynomial trends that simply provide a better visualization of temperature increase throughout the period between 1980 and 2100. Note: For the whole of New Zealand, only the A2 scenario increase is displayed on the graph, as the A1B and B1 increases are similar to the Marlborough region ones. 
Ainsi, selon le scénario pessimiste d'émission du GIEC, l'augmentation à l'échelle de la région devrait être supérieure de seulement $0,1^{\circ} \mathrm{C}$ par rapport à l'augmentation prévue au niveau national pour la fin du siècle. D'une manière générale, et selon ces premières projections à échelle fine, l'intensité de la hausse des températures prévue devrait être assez homogène sur l'ensemble du pays ; les plus grands écarts entre les régions ne devraient en effet pas dépasser $0,5^{\circ} \mathrm{C}$ (Reisinger et al., 2010). Cette amplitude est encore plus faible à l'échelle de la région de Marlborough, où les écarts thermiques ne dépassent pas $0,1^{\circ} \mathrm{C}$, quels que soient le scénario et la période.

L'analyse des écarts thermiques moyens mensuels à l'échelle de la région révèle des disparités significatives dans les amplitudes du réchauffement. Les mois d'octobre et novembre devraient connaître la hausse des températures la moins marquée selon les différents scénarios, entre 0,7 et $1,7^{\circ} \mathrm{C}$ respectivement pour le moyen terme et la fin du siècle. Comparativement, les mois de février, juillet et août devraient enregistrer la hausse la plus importante, de $1^{\prime}$ ordre de $1{ }^{\circ} \mathrm{C}$ à moyen terme et de $2,2^{\circ} \mathrm{C}$ à long terme (figure 13 ).

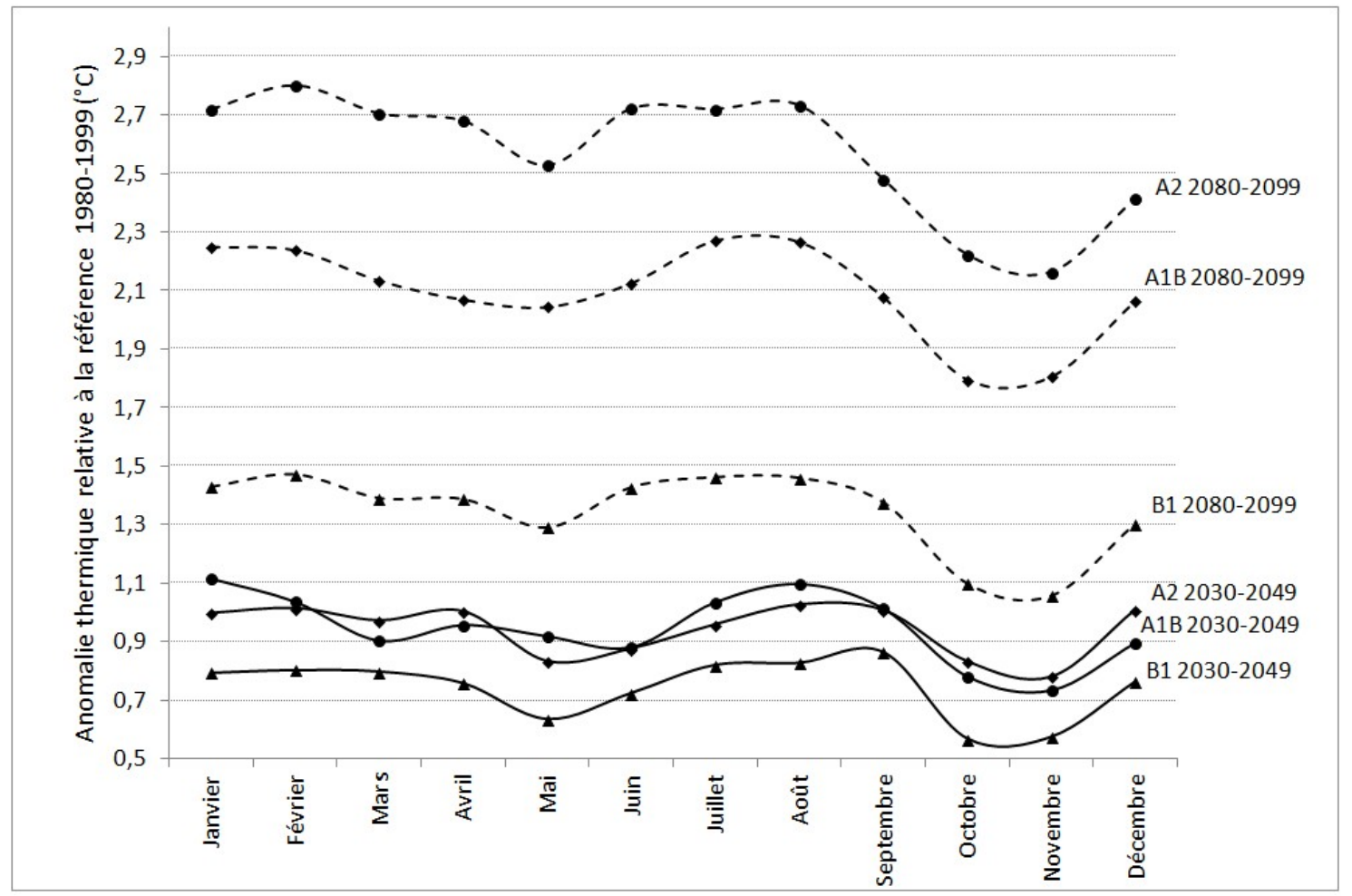

Figure 13: Anomalies thermiques moyennes mensuelles par rapport à la référence 1980-1999, calculées pour les trois scénarios du GIEC et les deux futures périodes (2030-2049 et 2080-2099) à l'échelle de la région de Marlborough. Average monthly temperature anomalies relative to the 1980-1999 reference, calculated for the three IPCC scenarios and the two future periods (2030-2049 and 2080-2099) across the Marlborough region.

\subsubsection{Une diminution significative du nombre de jours de gel}

Depuis 1970, suivant la tendance générale d'augmentation de la température, s'observe une diminution significative du nombre de jours de gel a l'échelle du pays (Wratt, 2009). Néanmoins, certaines zones, telle que la région de Marlborough, ont à l'inverse enregistré une augmentation de l'occurrence des gelées sur la même période. Le travail mené par Sturman et Quénol (2013) a par exemple mis en évidence une augmentation significative du nombre de jours de gel annuel et printanier entre 1941 et 2006 pour la station de Blenheim, ainsi qu'au niveau de la station de Christchurch, située $400 \mathrm{~km}$ au sud sur la côte est du pays. L'augmentation de la fréquence d'occurrence des types de temps anticycloniques (selon la 
classification de Kidson, 2000), combinée avec une recrudescence du flux de secteur sud ont respectivement favorisé une plus grande fréquence des gelées radiatives (lors de nuits claires et calmes) et advectives (arrivée d'une masse d'air plus froid provenant du sud). Les tendances prévues à moyen et long terme pour la région de Marlborough sont différentes de celles observées depuis une cinquantaine d'années. En effet, une diminution sensible du nombre de jours de gel annuel et printanier devrait être observée dès l'horizon 2030-2049.

Une première vue d'ensemble permet de constater que le principal facteur de variabilité de l'occurrence des gelées est la distance à l'océan, combinée à l'orographie (figure 14), des facteurs mis en évidence explicitement lors de l'analyse de la variabilité spatiale de la température entre 2008 et 2012 (cf. §2.1).

Figure 14 : Nombre de jours de gel moyen annuel simulé sur la période de référence 1980-1990, et projeté selon le scénario A1B du GIEC pour le moyen terme (2030-2049) et le long terme (20802099) à l'échelle de la région de Marlborough (cartes réalisées sous ArcMap ${ }^{\circledR}$ selon une méthode de Krigeage à partir des données calculées pour 300 points de grille). Mean annual number of days of frost modelled over the reference period 1980 1999, and expected for 2030-2049 and 2080-2099 according to the A1B IPCC scenario over the Marlborough region (maps built using ArcMap with a Kriging method from 300 grid points).

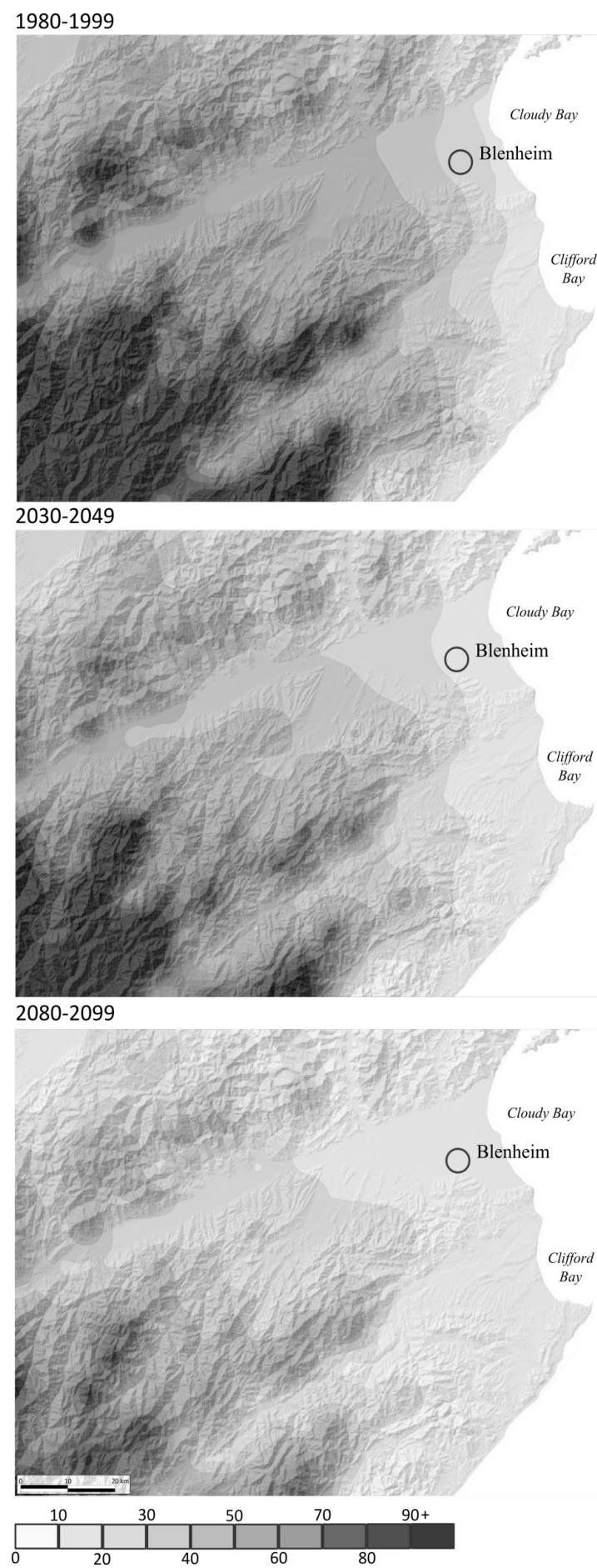


En revanche, tout en sachant qu'il s'agit de périodes différentes, il apparait également qu'une part de la variabilité locale de la température mise en évidence entre 2008 et 2012 ne se retrouve pas sur la période de référence 1980-1999. Entre autres, la plus grande occurrence de gelée qui s'observait au niveau de la station de Ward n'est pas visible sur la carte de référence. Il en va de même pour la vallée principale nord, où s'observait entre 2008 et 2012 moins d'occurrence de gelée au niveau de la station de Rapaura par rapport à Wratts Road et Blenheim Aero (figure 6). Cela peut s'expliquer, en partie, par le biais froid mis en évidence par l'évaluation sur la période 2006-2012 pour le point de grille le plus proche de la station (figure 3). De la même manière, les biais chauds calculés sur la même période aux stations de Wairau Valley et Waihopai Valley peuvent expliquer le plus faible nombre de jours de gel simulés sur la période de référence dans les vallées de Wairau et Waihopai, relativement à la période 2008-2012.

Tout en considérant ces biais potentiels associés à la simulation de référence, il semble évident que la diminution du nombre de jours de gel annuel à moyen et long terme à l'échelle de la région de Marlborough devrait être suffisamment importante pour engendrer une réduction significative du risque gélif pour les exploitants viticoles et ce, dès l'horizon 20302049. En moyenne, à l'échelle des deux principales vallées, devraient être observé une réduction de 10 jours de gelée à moyen terme, et de 20 jours d'ici la fin du siècle. Concernant les gelées printanières, la différence devrait surtout être effective en amont des vallées, notamment celle d'Awatere au sud de la région (figure 15).

Figure 15: Nombre de jours de gel au printemps (SON) simulé sur la période de référence 1980-1990 et selon le scénario A1B du GIEC pour le moyen terme (2030-2049) et le long terme (2080-2099) à l'échelle de la région de Marlborough (cartes réalisées sous ArcMap ${ }^{\circledR}$ selon une méthode de Krigeage à partir des données calculées pour 300 points de grille). Number of days of spring frost modelled over the reference period 1980-1999, and expected for 2030-2049 and 2080-2099 according to the A1B IPCC scenario over the Marlborough region (maps built using ArcMap with a Kriging method from 300 grid points).

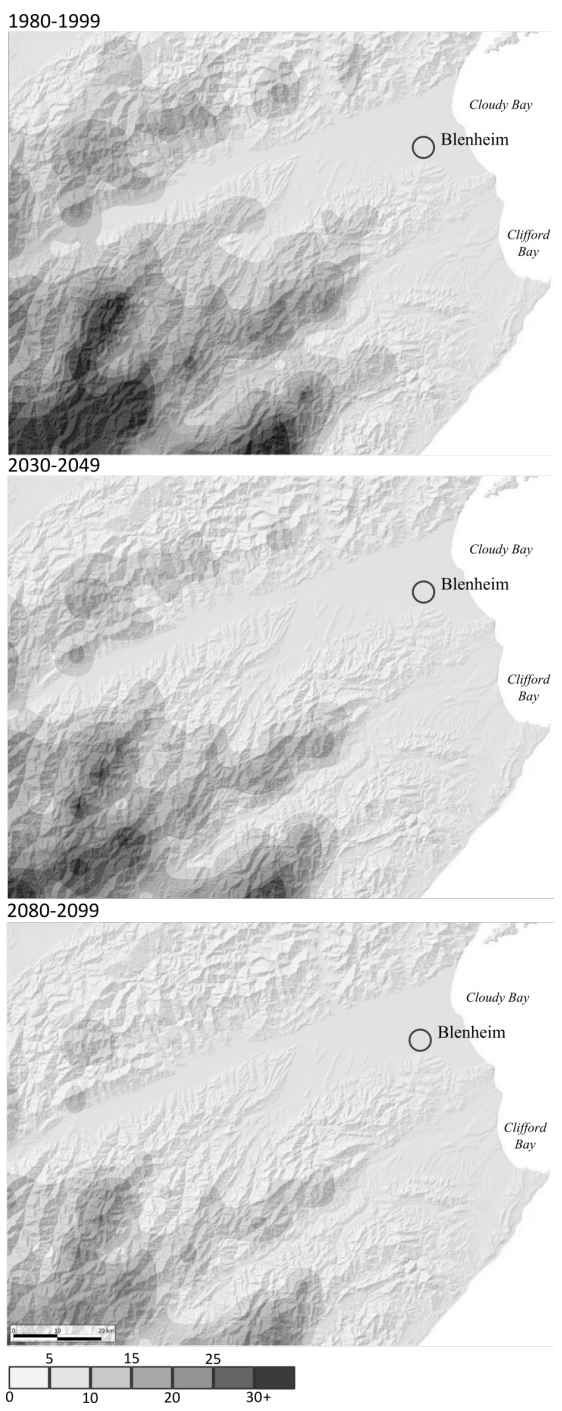




\subsubsection{Vers un changement des types de climats viticoles}

L'évaluation des indices bioclimatiques sur la période de référence 1980-1999 a révélé l'incertitude qui pouvait être associée à la reproduction des types de climats viticoles. Aussi, il ne s'agit pas d'interpréter les cartes obtenues de manière trop précise et définitive, sachant que la limite spatiale entre deux types de climats viticoles peut varier de manière sensible, notamment dans les parties les plus en amont de la région, où les biais calculés sont les plus importants.

Néanmoins, sachant ces incertitudes, les évolutions calculées pour les différents indices semblent assez sensibles pour affirmer qu'une modification des types de climats viticoles devrait pouvoir être observée dès 2030-2049 à l'échelle de la région. A moyen terme, le type de climat « frais » de Huglin devrait concerner l'ensemble de la vallée principale nord, ainsi que la partie aval de la vallée principale sud. A long terme, ces mêmes zones devraient être caractérisées par le type de climat tempéré de Huglin (figure 16), climat qui ne s'observe encore pas à l'échelle régionale.
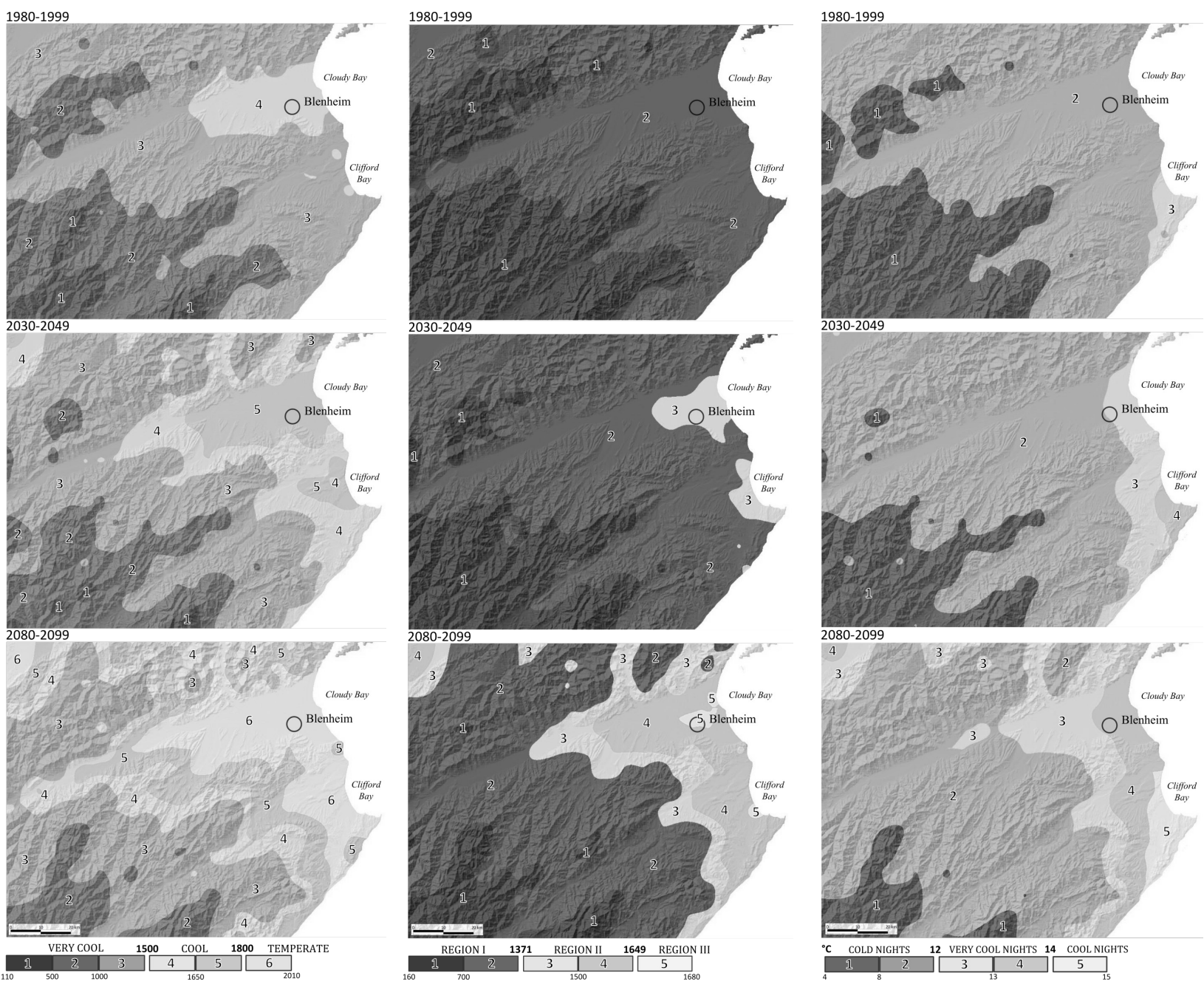

Figure 16 : à gauche) Valeurs d'indice de Huglin simulées sur la période de référence 1980-1990 et selon le scénario A1B du GIEC pour le moyen terme (2030-2049) et le long terme (2080-2099) à l'échelle de la région de Marlborough (cartes réalisées sous ArcMap ${ }^{\circledR}$ selon une méthode de Krigeage à partir des données calculées pour 300 points de grille). Au centre) Idem mais pour les valeurs d'indice de Winkler simulés. A droite) Idem mais pour les valeurs d'indice de fraicheur des nuits simulées. Left) Huglin index values modelled over the reference period 1980-1999 and expected for 2030-2049 and 2080-2099 according to the A1B IPCC scenario over the Marlborough region (maps built using ArcMap with a Kriging method from 300 grid points). Middle) Winkler index values modeled. Right) cool nights index values modeled. 
Selon l'évolution à moyen terme de l'indice de Winkler, les parties aval des deux principales vallées devraient passer de la région I à la région II. A long terme, le climat caractéristique de la région II selon Winkler devrait s'étendre en amont, alors que les parties les plus en aval pourraient basculer en région III (figure 16). Enfin, selon l'indice de fraicheur des nuits, les nuits très fraiches qui s'observent actuellement à la station de Cape Campbell, devraient concerner de manière progressive l'ensemble des parties aval des deux principales vallées (figure 16).

\section{Conclusion}

A l'échelle de la région de Marlborough est observée une forte variabilité spatiale des températures, conditionnée en grande partie par l'influence combinée des facteurs orographique et océanique. Notamment, l'augmentation significative de l'amplitude thermique et de l'occurrence des gelées (aussi bien annuelles que printanières) selon un gradient est-ouest, révèle cette forte variabilité régionale. Plus importante encore à considérer à l'échelle du vignoble, la variabilité thermique d'échelle locale, qui s'exprime sur des distances inférieures à 10 kilomètres, est particulièrement prononcée. C'est à cette échelle que s'exprime toute la complexité de la topographie très hétérogène de la région de Marlborough. Selon les zones, elle peut être à l'origine de situations d'abri vis-à-vis des vents synoptiques, ou à l'inverse, favoriser localement la turbulence et l'apport d'une nouvelle masse d'air en surface. Les écarts thermiques, notamment ceux relatifs aux températures minimales, peuvent alors être très importants d'une parcelle à l'autre. L'hétérogénéité spatiale des températures au niveau local engendre une vulnérabilité différentielle face au risque gélif entre des exploitants viticoles pourtant voisins. Cette variabilité locale est particulièrement difficile à appréhender. En effet, si des zones de plus fort risque peuvent aisément être identifiées à l'échelle de la région, surtout les zones les plus à l'ouest en amont des vallées, il reste délicat d'identifier ces même zones à risque au niveau local, tant les facteurs de variabilité spatiale et temporelle des températures sont complexes et nombreux à ce niveau d'échelle.

Le niveau d'incertitude quant aux facteurs de contrôle de cette variabilité, s'il grandit avec la descente d'échelle spatiale, grandit également avec le degré de projection pour l'avenir. Les évaluations des données d'observations virtuelles ont d'ailleurs mis en évidence les biais à associer aux simulations futures; des biais qui sont globalement limités sur la période de référence (connaissant le réseau d'observation utilisé pour élaborer l'interpolation), mais qui restent très importants à prendre en compte, étant donné que selon les zones, ils peuvent être équivalents à l'augmentation des températures prévue d'ici la fin du siècle. Néanmoins, avec ce degré d'incertitude inhérent à ces premières projections à échelle fine, l'augmentation prévue des températures devrait être assez significative pour engendrer une diminution notable du nombre de jours de gel dès l'horizon 2030-2049 à l'échelle du vignoble de Marlborough, réduisant ainsi de manière significative le risque gélif pour l'ensemble des exploitants viticoles.

La région (principalement dans sa partie est) devrait également connaître, d'ici une trentaine d'années, des changements dans les types de climats viticoles définis par les indices bioclimatiques de Huglin, Winkler et de fraicheur des nuits, très utilisés en viticulture. La région qui, à l'heure actuelle, est caractérisée par des types de climats plutôt froids pour la culture de la vigne (relativement aux valeurs des différents indices), devrait à moyen terme, mais surtout à long terme, être davantage caractérisée par des climats de types plus tempérés. Cela pourrait induire, entre autres, des modifications dans les stades de développement des cépages, ou encore, modifier les arômes du raisin (Bonnefoy et al., 2010 ; Quénol, 2012b), et par conséquent, les caractéristiques des vins produits.

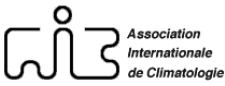


Les résultats issus de cette première analyse, menée à titre exploratoire, des projections pour le moyen terme et la fin du siècle, permettent d'apporter des premiers éléments de réponse quant aux potentiels impacts du changement climatique sur l'activité viti-vinicole de la région de Marlborough. Récemment, afin de mieux appréhender et comprendre les interactions entre les différents facteurs climatiques agissant sur les températures à une échelle locale, un réseau d'environ 70 capteurs thermiques a été mis en place à l'échelle du vignoble de Marlborough, selon des transects qui permettent de couvrir plusieurs sections d'une vallée. Les travaux en cours, relatifs à la modélisation à échelle fine pour l'ensemble de la Nouvelle-Zélande, combinés à ceux qui ont été menés dans le cadre du projet TERVICLIM/TERADCLIM, pourraient permettre de compléter et d'affiner les présents résultats. A terme, des adaptations pertinentes pourront éventuellement être mises en place auprès des exploitants viti-vinicoles de la région, et ainsi, permettre d'anticiper au mieux les potentielles modifications du climat local dans le contexte du changement climatique.

\section{Bibliographie}

AGENIS-NEVERS M., 2006 : Impacts du changement climatique sur les activités viti-vinicoles. Note technique $\mathrm{n}^{\circ} 3$, Observatoire National sur les Effets du Réchauffement Climatique, Paris, 20 pages.

Allaire G., Sylvander B., Belletti G., Marescotti A., Barjolle D., Thevenod-Motte E., TREGEAR A., 2005 : Les dispositifs français et européens de protection de la qualité et de l'origine dans le contexte de l'OMC - Justifications générales et contextes nationaux. Symposium international "Territoires et enjeux du développement régional", 21 pages.

BELTRANDO G., 1998 : Les gelées printanières en Champagne viticole - Quelques résultats obtenus à partir d'un réseau de stations automatiques. La Météorologie, $8^{\mathrm{ème}}$ série, 30-43.

BELTRANDO G., BRICHE E., 2010 : Changement climatique et viticulture en Champagne : du constat actuel aux prévisions du modèle ARPEGE-Climat sur l'évolution des températures pour le XXI ${ }^{\text {ème }}$ siècle. Echogéo, 14, 15 pages.

BONNEFOY C., 2013 : Observation et modélisation spatiale de la température dans les terroirs viticoles du Val de Loire dans le contexte du changement climatique. Thèse de doctorat de l'Université de Rennes 2, 309 pages.

Bonnefoy C., Quenol H., Planchon O., Barbeau G., 2010 : Températures et indices bioclimatiques dans le vignoble du Val de Loire dans un contexte de changement climatique. Echogéo, 14, 13 pages.

Briche E., Beltrando G., Somot S. Et QuÉnOl H., 2014 : Critical analysis of simulated daily temperature data from the ARPEGE-Climate model: application to climate change in the Champagne wine-producing region. Climatic Change. DOI: 10.1007/s10584-013-1044-5.

CHABIN J.-P., MADELIN M., BonNEFOY C., 2007 : Les vignobles beaunois face au réchauffement climatique. Actes du colloque international et pluridisciplinaire «Réchauffement climatique, quels impacts probables sur les vignobles? », Dijon et Beaune, 28-30 mars 2007, 13 pages.

COHEN S. J., 1990 : Bringing the global warming issue closer to home: the challenge of regional impact studies. Bullettin of the American Meteorological Society, 71, 520-526.

DUCHÊNE E., SCHNEIDER C., 2005 : Grapevine and climatic changes: a glance at the situation in Alsace. Agronomy for Sustainable Development, 25, 93-99.

IPCC, 2000 : Special Report on Emissions Scenarios. Nakicenovic N., Swart R. (eds), Cambridge University Press, Cambridge, United Kingdom, 104 pages. 
IPCC, 2007 : Climate Change: The Physical Science Basis. Summary for Policymakers, Contribution of the Working Group I to the Fourth Assessment of the Intergovernmental Panel on Climate Change, 19 pages.

IPCC, 2013 : Climate Change 2013. Publication du Volume 1 du 5e Rapport d'évaluation du GIEC, Cambridge Univ. Press, Cambridge, U.K.

Jones G. V., 2007 : Climate Change: Observations, Projections, and General Implications for Viticulture and Wine Production. Working Paper, Whitman College, Economics Department, 17 pages.

Jones G. V., Duchene E., Tomasi D., Yuste J., Braslavska O., Schultz H., Martinez C., Boso S., Langellier F., Perruchot C., Guimberteau G., 2005a : Changes in European Winegrape Phenology and relationships with climate. 54-61.

Jones G. V., White M. A., Cooper O. R., Storchmann K., 2005b : Climate change and global wine quality. Climatic Change, 73(3), 319-343.

KIDSON J. W., 2000 : An analysis of New Zealand synoptic types and their use in defining weather regimes. International Journal of Climatology. 20, 299-316.

Kim J.W., Chang J.T., BAKeR N.L., WiLKS D.S., GATES W.L., 1984 : The statistical problem of climate inversion : Determination of the relationship between local and larger-scale climate. Monthly Weather Review, 112, 2069-2077.

LAMB P., 1987 : On the development of regional climatic scenarios for policy oriented climatic impact assessment. Bulletin of American Meteorological Society, 68, 1116-1123.

MADELIN M., 2004 : l'aléa gélif printanier dans le vignoble marnais en Champagne: Modélisation spatiale aux échelles fines des températures minimales et des écoulements de l'air. Thèse de Doctorat de l'Université Paris VII, UMR Géographie-cité et UMR Prodig, 412 pages.

MADELIN M., Beltrando G., 2005 : Spatial interpolation-based mapping of the spring frost hazard in the Champagne vineyards. Meteorology, 12, 51-56.

MADELIN M., BOIS B., CHABIN J.-P., 2010 : Modification des conditions de maturation du raisin en Bourgogne viticole liée au réchauffement climatique. Echogéo, 14, 11 pages.

MARRE A., 2004 : Existe-t-il des terroirs viticoles en Champagne ? Revue Géographique de l'Est, volume 44/1-2, 17-30.

Mearns L. O., Giorgi F., Whetton P., Pabon D., Hulme M., Lal M., 2003 : Guidelines for use of climate scenarios developed from Regional Climate Model experiments. Data Distribution Centre of the Intergovernmental Panel on Climate Change, 38 pages.

MORAN W., 2000 : Culture et nature dans la géographie de l'industrie vinicole néo-zélandaise. Annales de Géographie, 109, n614-615, 525-551.

Mullan B., DAVID S. W., RENWICK J. A., 2002 : Transient model scenarios of climate change for New Zealand. Weather and Climate, 21, 3-34.

Mullan B., DEAn S., 2009 : AR4 climate model validation and scenarios for New Zealand. National Institute of Water \& Atmospheric Research Ldt, Wellington, New Zealand, 7 pages.

Mullan B., Wratt D. S., Dean S., Hollis M., Allan S., Williams T., Kenny G., 2008 : Climate Change Effects and Impacts Assessment: A Guidance Manual for Local Government in New Zealand, Second Edition. Ministry for the Environment, Wellington, 149 pages. 
NeEthling E., Barbeau G., Quenol H., Bonnefoy C., 2011 : Evolution du climat et de la composition des raisins des principaux cépages cultivés dans le val de Loire. Climatologie, 8, 77-90.

Powell S., STURMAn A., QuenOl H., 2011 : Changement climatique et variabilité spatiale du climat dans les vignobles de Nouvelle Zélande : l'exemple des vignobles de la région de Marlborough. Actes du XXIV ${ }^{\text {ème }}$ colloque de l'AIC, Rovereto, Italie, 477-482.

Quenol H., Monteiro A., Beltrando G., Maciel A., 2004 : Mesures climatiques aux échelles fines (météorologiques et agronomiques) et variabilité spatiale du gel printanier dans le vignoble de Vinho Verde (Portugal). Norois, 193, 117-132.

QUENOL H., 2012a : Observation et modélisation spatiale du climat aux échelles fines dans un contexte de changement climatique, Volume 1, Position et projet scientifique. Mémoire d'habilitation à diriger des recherches, Université Rennes 2, 103 pages.

QueNOL H., 2012b : Observation et modélisation du climat à l'échelle des terroirs viticoles. IX ${ }^{\text {ème }}$ Congrès International des Terroirs, Dijon/Reims, 25-29 juin 2012, 3, 15-17.

Reisinger A., Mullan, A. B., Manning M., Wratt D. W., 2010 : Global and local climate change scenarios to support adaptation in New Zealand. In: Climate change adaptation in New Zealand: Future scenarios and some sectoral perspectives, Nottage, R.A.C., Wratt D. S., Bornman, J. F., Jones K., New Zealand Climate Change Centre, Wellington, 26-43.

RoUSSET S., 2004 : Qualité et coordination économique dans les industries agroalimentaires - Analyse institutionnelle comparée de l'industrie du vin en Bourgogne, Californie et Nouvelle-Zélande. Thèse pour obtenir le grade de Docteur en Sciences Economique, Université de Bourgogne, 375 pages.

SCHULTZ H. R., 2000 : Climate change and viticulture: An European perspective on climatology, carbon dioxide and UV-B effects. Australian Journal of Grape and Wine Research, 6, 2-12.

SEGUIN B., 2007 : Le réchauffement climatique et ses conséquences pour la viticulture. Mission Changement climatique et effet de serre, INRA, 9 pages.

Seguin B., Garcia De Cortazar Atauri I., 2005 : Climate warming: consequences for viticulture and the notion of terroirs in Europe. Acta Horticulturae, 689, 61-71.

SLuYs S. L., 2006 : Climatic influences on the grapevine: A study of viticulture in the Waipara basin. Thesis submitted in partial fulfillment of the requirements for the Degree of Master of Science in Geography, University of Canterbury, 121 pages.

STURMAN A., QUENOL H., 2012 : Changes in atmospheric circulation and temperature trends in major vineyard regions of New Zealand. International Journal of Climatology, 33(12), 2609-2621. DOI: 10.1002/joc.3608.

Tait A., Henderson R., Turner R., Zheng X., 2006 : Thin plate smoothing spline interpolation of daily rainfall for $\mathrm{New}$ Zealand using a climatological rainfall surface. International Journal of Climatology, 26, 2097-2115.

Tondut J.-L., Laguet F., Deloire A., 2006 : Climat et viticulture : évolution des températures sur le département de l'Hérault : Un exemple de réchauffement climatique. Revue Française d'œnologie, 219, 1-14.

TONIETTO J., 1999 : Les macroclimats viticoles modiaux et l'influence du mesoclimat sur la typicité de la Syrah et du Muscat de Hambourg dans le sud de la France - Méthodologie de caractérisation. Thèse de Doctorat, Ecole Nationale Supérieure Agronomique de Montpellier, 236 pages. 
Van Leeuwen C., Garnier C., Agut C., Baculat B., Barbeau G., Besnard E., Bois B., Boursiquot J. M., Chuine I., Dessup T., DufourcQ T., Garcia-Cortazar I., Marguerit E., Monamy C., Koundouras S., Payan J. C., Parker A., Renouf V., Rodriguez-Lovelle B., Roby J. P., Tonietto J., Trambouze W., 2008: Heat requirements for grapevine varieties is essential information to adapt plant material in a changing climate. VII ${ }^{\mathrm{e} m e}$ Congrès International des terroirs viticoles, 6 pages.

WebB L., Whetton P. H., BARlow E. W. R., 2008 : Climate change and winegrape quality in Australia. Climate Research, 36, 99-111.

Wilby R. L., Charles S. P., Zorita E., Timbal B., Whetton P., Mearns L. O., 2004 : Guidelines for use of climate scenarios developed from statistical downscaling methods, 2-27.

WRATT D. S., 2009 : Climate change, climate variability, and the future. NIWA, Wellington, 57-59. 